\title{
A SENSITIVITY-BASED EXTRAPOLATION TECHNIQUE FOR THE NUMERICAL SOLUTION OF STATE-CONSTRAINED OPTIMAL CONTROL PROBLEMS $*, * *$
}

\author{
Michael HintermülleR ${ }^{1,2}$ AND IRWin YOUSEPT ${ }^{3}$
}

\begin{abstract}
Sensitivity analysis (with respect to the regularization parameter) of the solution of a class of regularized state constrained optimal control problems is performed. The theoretical results are then used to establish an extrapolation-based numerical scheme for solving the regularized problem for vanishing regularization parameter. In this context, the extrapolation technique provides excellent initializations along the sequence of reducing regularization parameters. Finally, the favorable numerical behavior of the new method is demonstrated and a comparison to classical continuation methods is provided.
\end{abstract}

Mathematics Subject Classification. 49M15, 49M37, 65K05, 90C33.

Received July 13, 2007. Revised January 28, 2009.

Published online July 2nd, 2009.

\section{INTRODUCTION}

The numerical treatment of optimal control problems for partial differential equations (PDEs) with pointwise state inequality constraints is challenging due to the measure-valuedness of the Lagrange multiplier associated with the state constraints; see [2] for an analytical assessment. A typical instance of such a problem is given by

$$
\left\{\begin{array}{l}
\text { minimize } J(u, y):=\frac{1}{2}\left\|y-y_{d}\right\|_{L^{2}(\Omega)}^{2}+\frac{\alpha}{2}\|u\|_{L^{2}(\Omega)}^{2} \\
\text { over }(u, y) \in L^{2}(\Omega) \times H_{0}^{1}(\Omega) \cap H^{2}(\Omega) \\
\text { subject to } A y=u \text { in } \Omega, \quad y=0 \text { on } \Gamma \\
y_{a} \leq y \leq y_{b} \quad \text { a.e. in } \Omega .
\end{array}\right.
$$

Keywords and phrases. Extrapolation, mixed control-state constraints, PDE-constrained optimization, semismooth Newton algorithm, sensitivity, state constraints.

* M.H. acknowledges support by the Austrian Ministry for Science and Education and the Austrian Science Fund FWF under START-program Y305 "Interfaces and Free Boundaries".

** I.Y. is supported by DFG Research Center Matheon, project C9: Numerical simulation and control of sublimation growth of semiconductor bulk single crystals.

1 Department of Mathematics Humboldt-University of Berlin, Unter den Linden 6, 10099 Berlin, Germany.

hint@math.hu-berlin.de

2 Institute of Mathematics and Scientific Computing, University of Graz, 8010 Graz, Austria.

3 Institut für Mathematik, Technische Universität Berlin, Str. des 17. Juni 136, 10623 Berlin, Germany.

yousept@math.tu-berlin.de 
where $\Omega \subset \mathbb{R}^{N}$ is a bounded and sufficiently regular domain, $A$ is a second order elliptic partial differential operator, and $y_{d}, \alpha, y_{a}, y_{b}$ are given data which will be specified shortly.

In order to have a numerical technique at hand for solving $(P)$ with stable iteration numbers as the mesh size of discretization is reduced, one can use an approach based on mixed control-state constraints as investigated in [12]. In fact, in order to overcome the measure-valuedness of the Lagrange multiplier associated with the pointwise inequality constraints in $(P)$ one adds $\epsilon u$ to $y$ in the set of pointwise inequality constraints and then uses the result in [12] which yields an $L^{2}$-property of the Lagrange multiplier associated with the modified set of constraints. The regular multiplier therefore represents a smooth approximation of the measure-valued quantity. Using this technique, $(P)$ becomes

$$
\left\{\begin{array}{l}
\operatorname{minimize} J(u, y):=\frac{1}{2}\left\|y-y_{d}\right\|_{L^{2}(\Omega)}^{2}+\frac{\alpha}{2}\|u\|_{L^{2}(\Omega)}^{2} \\
\text { over }(u, y) \in L^{2}(\Omega) \times H_{0}^{1}(\Omega) \\
\text { subject to } A y=u \text { in } \Omega, \quad y=0 \quad \text { on } \Gamma \\
y_{a} \leq \epsilon u+y \leq y_{b} \quad \text { a.e. in } \Omega .
\end{array}\right.
$$

Let $\left(\bar{y}_{\epsilon}, \bar{u}_{\epsilon}\right)$ denote the solution of $\left(P_{\epsilon}\right)$. For solving $\left(P_{\epsilon}\right)$ efficiently, in $[4,8]$ a semismooth Newton method (SSN) was proposed on a function space as well as on a discrete level. Besides the locally superlinear convergence, the mesh-independence of SSN for $\epsilon>0$ was established.

In view of $(P)$ one is interested in studying the behavior of the solution algorithm as $\epsilon \rightarrow 0$. For the numerics in the case of vanishing regularization parameter, however, in $[4,8]$ it turned out to be crucial to suitably tune $\epsilon$ as it tends to zero and, even more importantly, to initialize the algorithm for solving $\left(P_{\epsilon}\right)$ appropriately along the sequence. Ignoring these issues typically makes the numerical algorithm suffer from ill-conditioning which is usually reflected by large iteration numbers and reduced numerical solution accuracy.

In this note we focus on this latter point and propose an numerical approach based on an extrapolation technique in order to overcome the aforementioned problems. For this, we first need to study the quality of the dependence of $\left(\bar{y}_{\epsilon}, \bar{u}_{\epsilon}\right)$ on $\epsilon$. For instance, under a strict complementarity assumption we prove differentiability of the solution to $\left(P_{\epsilon}\right)$ with respect to $\epsilon$. Let $\left(\dot{y}_{\epsilon}, \dot{u}_{\epsilon}\right)$ denote the corresponding derivative. We then establish a system of sensitivity equations which characterize $\left(\dot{y}_{\epsilon}, \dot{u}_{\epsilon}\right)$ uniquely. Subsequently, the theoretical findings are employed in our numerical approach.

The remainder of the paper is organized as follows: in the rest of this section we detail the problem under investigation and settle the notation (Sect. 1.1), and we recall some results on $\left(P_{\epsilon}\right)$ (Sect. 1.2). The subsequent Section 2 is devoted to Lipschitz and differentiability properties of the solution to $\left(P_{\epsilon}\right)$ with respect to $\epsilon>0$. Then, in Section 3 we define our semismooth Newton-type solver based on extrapolation in $\epsilon$. We end this paper by a report on the numerical behavior including a comparison to a technique without extrapolation in Section 4. It turns out that our new method compares favorably to classical continuation methods without extrapolation.

\subsection{General assumptions and notation}

In connection with our model problem $(P)$, throughout this paper we assume that $\Omega$ is an open bounded domain in $\mathbb{R}^{N}, N \in\{2,3\}$, with sufficiently smooth boundary $\Gamma$. The upper and lower bounds $y_{a}, y_{b} \in \mathcal{C}(\bar{\Omega})$ on the state variable $y$ satisfy $y_{a}(x)<y_{b}(x)$ for all $x \in \bar{\Omega}$ and guarantee that the feasible set of $(P)$ is non-empty. Moreover, the desired state $y_{d} \in L^{2}(\Omega)$ and $\alpha>0$ are assumed to be fixed. By $u$ we denote the control variable. The second-order elliptic partial differential operator $A$ is defined by

$$
A y(x)=-\sum_{i, j=1}^{N} D_{i}\left(a_{i j}(x) D_{j} y(x)\right),
$$


where the coefficient functions $a_{i j} \in C^{0,1}(\bar{\Omega})$ satisfy the ellipticity condition

$$
\sum_{i, j=1}^{N} a_{i j}(x) \xi_{i} \xi_{j} \geq \theta\|\xi\|_{\mathbb{R}^{N}}^{2} \quad \forall(\xi, x) \in \mathbb{R}^{N} \times \bar{\Omega}
$$

for some constant $\theta>0$. Furthermore, $A^{\star}$ stands for the associated adjoint operator. By $G$ we denote the solution operator $G: L^{2}(\Omega) \rightarrow H_{0}^{1}(\Omega) \cap H^{2}(\Omega)$ that assigns to every $u \in L^{2}(\Omega)$ the solution $y=y(u) \in$ $H_{0}^{1}(\Omega) \cap H^{2}(\Omega)$ of the state equation

$$
A y=u \text { in } \Omega, \quad y=0 \text { on } \Gamma .
$$

We set $S=\imath_{0} G$, where $\imath_{0}$ denotes the compact embedding operator from $H^{1}(\Omega)$ to $L^{2}(\Omega)$.

Using the above notation and assumptions, the regularized problem $\left(P_{\epsilon}\right)$ can be expressed as follows:

$$
\left\{\begin{array}{l}
\text { minimize } f(u):=\frac{1}{2}\left\|S u-y_{d}\right\|_{L^{2}(\Omega)}^{2}+\frac{\alpha}{2}\|u\|_{L^{2}(\Omega)}^{2} \quad \text { over } u \in L^{2}(\Omega) \\
\text { subject to } y_{a} \leq(S+\epsilon I) u \leq y_{b} \quad \text { a.e. in } \Omega,
\end{array}\right.
$$

where $I$ denotes the identity operator in $L^{2}(\Omega)$. In [10] the name Lavrentiev regularized problem was coined for $\left(P_{\epsilon}\right)$.

\subsection{Standard results}

For every $\epsilon>0$ standard arguments guarantee the existence of a unique solution of $\left(P_{\epsilon}\right)$. Throughout the paper we denote this solution by $\bar{u}_{\epsilon}$ with associated state $\bar{y}_{\epsilon}$. As in [10], first order optimality of $\left(\bar{y}_{\epsilon}, \bar{u}_{\epsilon}\right)$ can be characterized as follows:

Theorem 1.1 (first-order optimal conditions for $\left.\left(P_{\epsilon}\right)\right)$. The pair $\left(\bar{y}_{\epsilon}, \bar{u}_{\epsilon}\right)$ is optimal for $\left(P_{\epsilon}\right)$ if and only if there exist an adjoint state $p_{\epsilon} \in H_{0}^{1}(\Omega) \cap H^{2}(\Omega)$ and Lagrange multipliers $\mu_{\epsilon}^{a}, \mu_{\epsilon}^{b} \in L^{2}(\Omega)$ such that

$$
\begin{aligned}
& A \bar{y}_{\epsilon}=\bar{u}_{\epsilon} \quad \text { in } \Omega, \quad \bar{y}_{\epsilon}=0 \quad \text { on } \Gamma, \\
& A^{\star} p_{\epsilon}=\bar{y}_{\epsilon}-y_{d}+\mu_{\epsilon}^{b}-\mu_{\epsilon}^{a} \quad \text { in } \Omega, \quad p_{\epsilon}=0 \quad \text { on } \Gamma, \\
& p_{\epsilon}+\alpha \bar{u}_{\epsilon}-\epsilon\left(\mu_{\epsilon}^{a}-\mu_{\epsilon}^{b}\right)=0, \\
& \epsilon \bar{u}_{\epsilon}+\bar{y}_{\epsilon} \geq y_{a}, \quad \mu_{\epsilon}^{a} \geq 0, \quad\left(\mu_{\epsilon}^{a}, \epsilon \bar{u}_{\epsilon}+\bar{y}_{\epsilon}-y_{a}\right)_{L^{2}(\Omega)}=0, \\
& \epsilon \bar{u}_{\epsilon}+\bar{y}_{\epsilon} \leq y_{b}, \quad \mu_{\epsilon}^{b} \geq 0, \quad\left(\mu_{\epsilon}^{b}, \epsilon \bar{u}_{\epsilon}+\bar{y}_{\epsilon}-y_{b}\right)_{L^{2}(\Omega)}=0 .
\end{aligned}
$$

Using the maximum operator, the complementarity system (1.4)-(1.5) can be equivalently expressed as

$$
\begin{aligned}
& \mu_{\epsilon}^{a}=\max \left(0, \mu_{\epsilon}^{a}-\mu_{\epsilon}^{b}+\gamma\left(y_{a}-\epsilon \bar{u}_{\epsilon}-\bar{y}_{\epsilon}\right)\right), \\
& \mu_{\epsilon}^{b}=\max \left(0, \mu_{\epsilon}^{b}-\mu_{\epsilon}^{a}+\gamma\left(\epsilon \bar{u}_{\epsilon}+\bar{y}_{\epsilon}-y_{b}\right)\right),
\end{aligned}
$$


with an arbitrarily fixed $\gamma>0$, cf. [5,13]. Note that $\gamma$ acts as a weight between the inequality constraints for the primal variable and the corresponding dual variables or Lagrange multipliers. For the choice $\gamma:=\alpha / \epsilon^{2}$ in (1.6)-(1.7) and using (1.3), a short computation yields

$$
\begin{aligned}
\mu_{\epsilon}^{a} & =\max \left(0, \frac{1}{\epsilon} p_{\epsilon}+\frac{\alpha}{\epsilon^{2}}\left(y_{a}-\bar{y}_{\epsilon}\right)\right), \\
\mu_{\epsilon}^{b} & =\max \left(0,-\frac{1}{\epsilon} p_{\epsilon}+\frac{\alpha}{\epsilon^{2}}\left(\bar{y}_{\epsilon}-y_{b}\right)\right) .
\end{aligned}
$$

This latter system will be useful in our subsequent analysis.

Finally, we mention that in [11], Theorem 3.3, the strong convergence in $L^{2}(\Omega)$ of $\bar{u}_{\epsilon}$ to $\bar{u}$, the optimal control of $(P)$, is proven. From the results in [9], Lemma 2.4, the weak* convergence in $C(\bar{\Omega})^{*}$ as $\epsilon \rightarrow 0$ of $\mu_{\epsilon}^{a}$ and $\mu_{\epsilon}^{b}$ to $\bar{\mu}^{a}$ and $\bar{\mu}^{b}$, the multipliers associated with the pointwise state constraints at the solution of $(P)$, can be inferred. Further, in [4] the Hölder continuity (with exponent $\frac{1}{2}$ ) of $\bar{u}_{\epsilon}$ with respect to $\epsilon>0$ is established.

\section{Regularity of solutions to $\left(P_{\epsilon}\right)$}

As pointed out in the introduction, one of our main goals is to establish Lipschitz continuity and differentiability of the mapping $\epsilon \mapsto \bar{y}_{\epsilon}$. For this purpose, the transformation of $\left(P_{\epsilon}\right)$ into an associated minimization problem with pure control constraints will be useful.

We start our investigation by considering the operator $(\epsilon I+S)$. It is well known that the linear operator $S=\imath_{0} G: L^{2}(\Omega) \rightarrow L^{2}(\Omega)$ is positive-definite. For this reason, the equation

$$
(\epsilon I+S) z=0
$$

admits only the trivial solution $z=0$. Thus, by the Fredholm alternative, the compactness property of $S$ ensures the existence of the inverse operator $(\epsilon I+S)^{-1}$. Setting $(\epsilon I+S)^{-1} v=u$, or equivalently $u=\frac{1}{\epsilon}\left(v-\imath_{0} y(u)\right)$ in $\left(P_{\epsilon}\right)$, we transform $\left(P_{\epsilon}\right)$ into the following optimal control problem with (pointwise) box constraints imposed on the new control variable $v$ :

$$
\left\{\begin{array}{l}
\operatorname{minimize} f_{\epsilon}(v):=\frac{1}{2}\left\|S_{\epsilon} v-y_{d}\right\|_{L^{2}(\Omega)}^{2}+\frac{\alpha}{2 \epsilon^{2}}\left\|v-S_{\epsilon} v\right\|_{L^{2}(\Omega)}^{2} \quad \text { over } v \in L^{2}(\Omega) \\
\text { subject to } y_{a} \leq v \leq y_{b} \quad \text { a.e. } \operatorname{in} \Omega .
\end{array}\right.
$$

Here we use $S_{\epsilon}=\imath_{0} G_{\epsilon}$, where the operator $G_{\epsilon}$ assigns to each $v \in L^{2}(\Omega)$ the solution $y_{\epsilon}(v)=y_{\epsilon} \in H_{0}^{1}(\Omega) \cap H^{2}(\Omega)$ of the following elliptic equation:

$$
A y_{\epsilon}+\frac{1}{\epsilon} y_{\epsilon}=\frac{1}{\epsilon} v \quad \text { in } \Omega, \quad y_{\epsilon}=0 \quad \text { on } \Gamma .
$$

Notice that the underlying ideas of converting $\left(P_{\epsilon}\right)$ into $\left(P_{\epsilon}^{v}\right)$ and considering the state equation in the form $\left(E_{\epsilon}\right)$ were originally introduced in $[10,11]$. In what follows, we denote the unique solution of $\left(P_{\epsilon}^{v}\right)$ by $\bar{v}_{\epsilon}$ with associated optimal state $\bar{y}_{\epsilon}$. Obviously, $\left(P_{\epsilon}^{v}\right)$ and $\left(P_{\epsilon}\right)$ are equivalent, i.e., $\bar{v}_{\epsilon}$ solves $\left(P_{\epsilon}^{v}\right)$ if and only if $\bar{u}_{\epsilon}=(\epsilon I+S)^{-1} \bar{v}_{\epsilon}$ is the optimal solution of $\left(P_{\epsilon}\right)$. We use the auxiliary problem $\left(P_{\epsilon}^{v}\right)$ in the following analysis.

The first derivative of $f_{\epsilon}$ at $v$ in an arbitrary direction $s \in L^{2}(\Omega)$ is given by

$$
f_{\epsilon}^{\prime}(v) s=\left(S_{\epsilon} v-y_{d}+\frac{\alpha}{\epsilon^{2}}\left(S_{\epsilon} v-v\right), S_{\epsilon} s\right)_{L^{2}(\Omega)}+\frac{\alpha}{\epsilon^{2}}\left(v-S_{\epsilon} v, s\right)_{L^{2}(\Omega)} .
$$

By standard arguments, this can be equivalently expressed as

$$
f_{\epsilon}^{\prime}(v)=q_{\epsilon}(v)-\frac{\alpha}{\epsilon^{2}} y_{\epsilon}(v)+\frac{\alpha}{\epsilon^{2}} v,
$$


where the adjoint state $q_{\epsilon}=q_{\epsilon}(v) \in H_{0}^{1}(\Omega) \cap H^{2}(\Omega)$ is defined as the solution of the following adjoint equation:

$$
A^{\star} q_{\epsilon}+\frac{1}{\epsilon} q_{\epsilon}=\frac{1}{\epsilon}\left(y_{\epsilon}(v)-y_{d}+\frac{\alpha}{\epsilon^{2}}\left(y_{\epsilon}(v)-v\right)\right) \quad \text { in } \Omega, \quad q_{\epsilon}=0 \quad \text { on } \Gamma .
$$

\subsection{Lipschitz continuity}

Next we establish a Lipschitz property of $y_{\epsilon}$ and various other quantities. Since one might by interested in $\left(P_{\epsilon}\right)$ independently of $(P)$, we reduce the regularity requirements on, e.g., $\Omega$, as we do not assume to have $H^{2}(\Omega)$-regularity of the states and adjoints pertinent to $\left(P_{\epsilon}\right)$. We only consider a Lipschitz-domain $\Omega \subset \mathbb{R}^{N}$ with $N \in\{2,3\}$ which is sufficient to obtain $H^{1}(\Omega) \cap \mathcal{C}(\bar{\Omega})$-regularity of the solutions to the adjoint and state equations (see the elliptic regularity results in [1]).

In what follows, let $\mathcal{D}=\left(\epsilon_{l}, \epsilon_{u}\right) \subset \mathbb{R}_{++}$with $0<\epsilon_{l}<\epsilon_{u}$.

Lemma 2.1. There exists a positive real number $c_{s}\left(\epsilon_{l}\right)$ such that

$$
\left\|y_{\epsilon_{1}}(v)-y_{\epsilon_{2}}(v)\right\|_{H_{0}^{1}(\Omega) \cap \mathcal{C}(\bar{\Omega})} \leq c_{s}\left(\epsilon_{l}\right)\|v\|_{L^{2}(\Omega)}\left|\epsilon_{1}-\epsilon_{2}\right|
$$

for all $v \in L^{2}(\Omega)$ and $\epsilon_{i} \in \mathcal{D}, i=1,2$.

Proof. We note that standard elliptic regularity estimates yield a constant $c>0$ independent of $\epsilon$ and $v$ such that

$$
\left\|y_{\epsilon}(v)\right\|_{H_{0}^{1}(\Omega) \cap \mathcal{C}(\bar{\Omega})} \leq c\left\|\frac{1}{\epsilon} v\right\|_{L^{2}(\Omega)}
$$

for all $v \in L^{2}(\Omega)$ and $\epsilon \in \mathcal{D}$. Now, let $\epsilon_{i} \in \mathcal{D}, i=1,2$, and $v \in L^{2}(\Omega)$. By definition, for $i=1,2$ the states $y_{\epsilon_{i}}(v)=y_{\epsilon_{i}}$ satisfy

$$
A y_{\epsilon_{i}}+\frac{1}{\epsilon_{i}} y_{\epsilon_{i}}=\frac{1}{\epsilon_{i}} v \quad \text { in } \Omega, \quad y_{\epsilon_{i}}=0 \quad \text { on } \Gamma \text {. }
$$

From this we infer

$$
A\left(y_{\epsilon_{1}}-y_{\epsilon_{2}}\right)+\frac{1}{\epsilon_{1}}\left(y_{\epsilon_{1}}-y_{\epsilon_{2}}\right)=\left(\frac{1}{\epsilon_{1}}-\frac{1}{\epsilon_{2}}\right)\left(v-y_{\epsilon_{2}}\right) \quad \text { in } \Omega, \quad y_{\epsilon_{1}}-y_{\epsilon_{2}}=0 \quad \text { on } \Gamma,
$$

and hence due to $(2.3)$

$$
\begin{aligned}
\left\|y_{\epsilon_{1}}(v)-y_{\epsilon_{2}}(v)\right\|_{H_{0}^{1}(\Omega) \cap \mathcal{C}(\bar{\Omega})} & \leq\left|\frac{1}{\epsilon_{1}}-\frac{1}{\epsilon_{2}}\right| c\left\|v-y_{\epsilon_{2}}(v)\right\|_{L^{2}(\Omega)} \\
& \leq\left|\epsilon_{1}-\epsilon_{2}\right| \frac{c}{\epsilon_{l}^{2}}\left(\|v\|_{L^{2}(\Omega)}+\left\|y_{\epsilon_{2}}(v)\right\|_{L^{2}(\Omega)}\right) \\
& \leq\left|\epsilon_{1}-\epsilon_{2}\right| \frac{c}{\epsilon_{l}^{2}}\left(1+\frac{c}{\epsilon_{l}}\right)\|v\|_{L^{2}(\Omega)} .
\end{aligned}
$$

Setting $c_{s}\left(\epsilon_{l}\right):=\frac{c}{\epsilon_{l}^{2}}\left(1+\frac{c}{\epsilon_{l}}\right)$ yields the assertion.

We proceed by proving the Lipschitz continuity of the adjoint states $q_{\epsilon}$.

Lemma 2.2. There exists a positive real number $c_{q}(\mathcal{D})$ such that

$$
\left\|q_{\epsilon_{1}}(v)-q_{\epsilon_{2}}(v)\right\|_{H_{0}^{1}(\Omega) \cap \mathcal{C}(\bar{\Omega})} \leq c_{q}(\mathcal{D})\left(\|v\|_{L^{2}(\Omega)}+1\right)\left|\epsilon_{1}-\epsilon_{2}\right|,
$$

for all $v \in L^{2}(\Omega)$ and $\epsilon_{1}, \epsilon_{2} \in \mathcal{D}$. 
Proof. An argument analogous to the one in the proof of Lemma 2.1 implies that there exists a constant $c(\mathcal{D})>0$ independent of $\epsilon$ and $v$ such that

$$
\left\|q_{\epsilon}(v)\right\|_{H_{0}^{1}(\Omega) \cap \mathcal{C}(\bar{\Omega})} \leq c(\mathcal{D})\left(\|v\|_{L^{2}(\Omega)}+1\right)
$$

for all $\epsilon \in \mathcal{D}$ and $v \in L^{2}(\Omega)$. Let $\epsilon_{1}, \epsilon_{2} \in \mathcal{D}$. By definition $q_{\epsilon_{1}}(v)=q_{\epsilon_{1}}$ and $q_{\epsilon_{2}}(v)=q_{\epsilon_{2}}$ solve, for $i=1,2$,

$$
A^{\star} q_{\epsilon_{i}}+\frac{1}{\epsilon_{i}} q_{\epsilon_{i}}=\frac{1}{\epsilon_{i}}\left(y_{\epsilon_{i}}(v)-y_{d}+\frac{\alpha}{\epsilon_{i}^{2}}\left(y_{\epsilon_{i}}(v)-v\right)\right) \quad \text { in } \Omega, \quad q_{\epsilon_{i}}=0 \quad \text { on } \Gamma .
$$

Hence, the difference $q_{\epsilon_{1}}-q_{\epsilon_{2}}$ satisfies

$$
A^{\star}\left(q_{\epsilon_{1}}-q_{\epsilon_{2}}\right)+\frac{1}{\epsilon_{1}}\left(q_{\epsilon_{1}}-q_{\epsilon_{2}}\right)=r \quad \text { in } \Omega, \quad q_{\epsilon_{1}}-q_{\epsilon_{2}}=0 \quad \text { on } \Gamma,
$$

where

$$
\begin{aligned}
r=\frac{1}{\epsilon_{1}}\left(y_{\epsilon_{1}}(v)-y_{\epsilon_{2}}(v)\right) & +\left(\frac{1}{\epsilon_{1}}-\frac{1}{\epsilon_{2}}\right)\left(y_{\epsilon_{2}}(v)-y_{d}\right)+\frac{\alpha}{\epsilon_{1}^{3}}\left(y_{\epsilon_{1}}(v)-y_{\epsilon_{2}}(v)\right) \\
& +\left(\frac{\alpha}{\epsilon_{1}^{3}}-\frac{\alpha}{\epsilon_{2}^{3}}\right)\left(y_{\epsilon_{2}}(v)-v\right)+\left(\frac{1}{\epsilon_{2}}-\frac{1}{\epsilon_{1}}\right) q_{\epsilon_{2}} .
\end{aligned}
$$

Since $\left|\epsilon_{2}^{3}-\epsilon_{1}^{3}\right|=\left|\left(\epsilon_{2}^{2}+\epsilon_{1}^{2}+\epsilon_{2} \epsilon_{1}\right)\left(\epsilon_{2}-\epsilon_{1}\right)\right| \leq 3 \epsilon_{u}^{2}\left|\epsilon_{2}-\epsilon_{1}\right|$, Lemma 2.1 and (2.4) yield a constant $c_{q}(\mathcal{D})>0$ independent of $\epsilon_{1}, \epsilon_{2}$ such that

$$
\left\|q_{\epsilon_{1}}(v)-q_{\epsilon_{2}}(v)\right\|_{H_{0}^{1}(\Omega) \cap \mathcal{C}(\bar{\Omega})} \leq\|r\|_{L^{2}(\Omega)} \leq c_{q}(\mathcal{D})\left|\epsilon_{1}-\epsilon_{2}\right|\left(\|v\|_{L^{2}(\Omega)}+1\right),
$$

which ends the proof.

For arguing the Lipschitz continuity of the controls, we require uniform positive definiteness of $f_{\epsilon}^{\prime \prime}$, which we establish next.

Lemma 2.3. There exists a positive real number $\delta(\mathcal{D})$ such that for all $\epsilon \in \mathcal{D}$, we have

$$
f_{\epsilon}^{\prime \prime}(v) s^{2} \geq \delta(\mathcal{D})\|s\|_{L^{2}(\Omega)}^{2} \quad \forall v, s \in L^{2}(\Omega) .
$$

Proof. Let $v \in L^{2}(\Omega)$ and $\epsilon \in \mathcal{D}$ be arbitrarily fixed. Then, it holds by definition that $f_{\epsilon}(v)=f\left((S+\epsilon I)^{-1} v\right)$. Hence, for an arbitrary direction $s \in L^{2}(\Omega)$, the chain rule implies

$$
\begin{aligned}
f_{\epsilon}^{\prime \prime}(v) s^{2} & =f^{\prime \prime}\left((S+\epsilon I)^{-1} v\right)\left((S+\epsilon I)^{-1} s\right)^{2} \\
& =\left(S(S+\epsilon I)^{-1} s, S(S+\epsilon I)^{-1} s\right)_{L^{2}(\Omega)}+\alpha\left((S+\epsilon I)^{-1} s,(S+\epsilon I)^{-1} s\right)_{L^{2}(\Omega)} \\
& \geq \alpha\left((S+\epsilon I)^{-1} s,(S+\epsilon I)^{-1} s\right)_{L^{2}(\Omega)} \\
& =\alpha\left\|(S+\epsilon I)^{-1} s\right\|_{L^{2}(\Omega)}^{2} \\
& \geq \frac{\alpha}{\|S+\epsilon I\|_{L^{2}, L^{2}}^{2}}\|s\|_{L^{2}(\Omega)}^{2} \\
& \geq \frac{\alpha}{\left(\|S\|_{L^{2}, L^{2}}+\epsilon_{u}\right)^{2}}\|s\|_{L^{2}(\Omega)}^{2} .
\end{aligned}
$$

Defining $\delta(\mathcal{D}):=\alpha /\left(\|S\|_{L^{2}, L^{2}}+\epsilon_{u}\right)^{2}$, the lemma is verified. 
Theorem 2.1. The mapping $\mathbf{v}: \mathcal{D} \rightarrow L^{2}(\Omega), \epsilon \mapsto \bar{v}_{\epsilon}$, is Lipschitz-continuous.

Proof. Suppose that $\epsilon_{1}, \epsilon_{2} \in \mathcal{D}$. By definition $\mathbf{v}\left(\epsilon_{1}\right)=\bar{v}_{\epsilon_{1}}$ and $\mathbf{v}\left(\epsilon_{2}\right)=\bar{v}_{\epsilon_{2}}$ solve $\left(P_{\epsilon_{1}}^{v}\right)$ and $\left(P_{\epsilon_{2}}^{v}\right)$, respectively. Hence, first order optimality yields

$$
\begin{aligned}
& f_{\epsilon_{1}}^{\prime}\left(\bar{v}_{\epsilon_{1}}\right)\left(v-\bar{v}_{\epsilon_{1}}\right) \geq 0 \quad \forall v \in V_{\mathrm{ad}}, \\
& f_{\epsilon_{2}}^{\prime}\left(\bar{v}_{\epsilon_{2}}\right)\left(v-\bar{v}_{\epsilon_{2}}\right) \geq 0 \quad \forall v \in V_{\mathrm{ad}},
\end{aligned}
$$

where $V_{\mathrm{ad}}=\left\{v \in L^{2}(\Omega) \mid y_{a} \leq v \leq y_{b}\right\}$. Since $\bar{v}_{\epsilon_{1}}$ and $\bar{v}_{\epsilon_{2}}$ are feasible, i.e., $\bar{v}_{\epsilon_{1}}, \bar{v}_{\epsilon_{2}} \in V_{\mathrm{ad}}$, the inequalities above imply

which is equivalent to

$$
\left(f_{\epsilon_{1}}^{\prime}\left(\bar{v}_{\epsilon_{1}}\right)-f_{\epsilon_{2}}^{\prime}\left(\bar{v}_{\epsilon_{2}}\right)\right)\left(\bar{v}_{\epsilon_{2}}-\bar{v}_{\epsilon_{1}}\right) \geq 0
$$

$$
\left(f_{\epsilon_{1}}^{\prime}\left(\bar{v}_{\epsilon_{1}}\right)-f_{\epsilon_{1}}^{\prime}\left(\bar{v}_{\epsilon_{2}}\right)+f_{\epsilon_{1}}^{\prime}\left(\bar{v}_{\epsilon_{2}}\right)-f_{\epsilon_{2}}^{\prime}\left(\bar{v}_{\epsilon_{2}}\right)\right)\left(\bar{v}_{\epsilon_{2}}-\bar{v}_{\epsilon_{1}}\right) \geq 0
$$

Since $f_{\epsilon_{1}}^{\prime}$ is Fréchet-differentiable, there exists $\tilde{v} \in L^{2}(\Omega)$ such that

$$
\left(f_{\epsilon_{1}}^{\prime}\left(\bar{v}_{\epsilon_{1}}\right)-f_{\epsilon_{1}}^{\prime}\left(\bar{v}_{\epsilon_{2}}\right)\right)\left(\bar{v}_{\epsilon_{2}}-\bar{v}_{\epsilon_{1}}\right)=-f_{\epsilon_{1}}^{\prime \prime}(\tilde{v})\left(\bar{v}_{\epsilon_{2}}-\bar{v}_{\epsilon_{1}}\right)^{2} .
$$

Then (2.7) yields

$$
\left(f_{\epsilon_{1}}^{\prime}\left(\bar{v}_{\epsilon_{2}}\right)-f_{\epsilon_{2}}^{\prime}\left(\bar{v}_{\epsilon_{2}}\right)\right)\left(\bar{v}_{\epsilon_{2}}-\bar{v}_{\epsilon_{1}}\right) \geq f_{\epsilon_{1}}^{\prime \prime}(\tilde{v})\left(\bar{v}_{\epsilon_{2}}-\bar{v}_{\epsilon_{1}}\right)^{2}
$$

and further

$$
\begin{aligned}
& \left(\left\|q_{\epsilon_{1}}\left(\bar{v}_{\epsilon_{2}}\right)-q_{\epsilon_{2}}\left(\bar{v}_{\epsilon_{2}}\right)\right\|_{L^{2}(\Omega)}+\frac{\alpha}{\epsilon_{1}^{2}}\left\|y_{\epsilon_{1}}\left(\bar{v}_{\epsilon_{2}}\right)-y_{\epsilon_{2}}\left(\bar{v}_{\epsilon_{2}}\right)\right\|_{L^{2}(\Omega)}+\right. \\
& \left.\frac{\alpha}{\epsilon_{1}^{2} \epsilon_{2}^{2}}\left|\epsilon_{1}-\epsilon_{2}\right| \cdot\left|\epsilon_{1}+\epsilon_{2}\right|\left(\left\|y_{\epsilon_{2}}\left(\bar{v}_{\epsilon_{2}}\right)\right\|_{L^{2}(\Omega)}+\left\|\bar{v}_{\epsilon_{2}}\right\|_{L^{2}(\Omega)}\right)\right)\left\|\bar{v}_{\epsilon_{2}}-\bar{v}_{\epsilon_{1}}\right\|_{L^{2}(\Omega)} \geq f_{\epsilon_{1}}^{\prime \prime}(\tilde{v})\left(\bar{v}_{\epsilon_{2}}-\bar{v}_{\epsilon_{1}}\right)^{2},
\end{aligned}
$$

where we used (2.1). Due to Lemmas 2.1-2.3, there exists a constant $c(\mathcal{D})>0$ independent of $\epsilon_{1}, \epsilon_{2}$ such that

$$
c(\mathcal{D})\left|\epsilon_{2}-\epsilon_{1}\right|\left(\left\|\bar{v}_{\epsilon_{2}}\right\|_{L^{2}(\Omega)}+1\right) \geq\left\|\bar{v}_{\epsilon_{2}}-\bar{v}_{\epsilon_{1}}\right\|_{L^{2}(\Omega)} .
$$

Notice that the operator $\mathbf{v}(\cdot)$ is uniformly bounded in $L^{\infty}(\Omega)$, as $\bar{v}_{\epsilon} \in V_{\text {ad }}$ for all $\epsilon \in \mathbb{R}_{++}$. This together with (2.9) proves the assertion.

We have the following immediate corollaries establishing the Lipschitz continuity of $\bar{y}_{\epsilon}$ and $\bar{u}_{\epsilon}$.

Corollary 2.1. The mapping $\epsilon \mapsto \bar{y}_{\epsilon}$ is Lipschitz-continuous from $\mathcal{D}$ to $H_{0}^{1}(\Omega) \cap \mathcal{C}(\bar{\Omega})$.

Proof. Theorem 2.1 and Lemma 2.1 guarantee the existence of a constant $c(\mathcal{D})>0$ independent of $\epsilon_{1}, \epsilon_{2} \in \mathcal{D}$ such that

$$
\left\|\bar{y}_{\epsilon_{1}}-\bar{y}_{\epsilon_{2}}\right\|_{H_{0}^{1}(\Omega) \cap \mathcal{C}(\bar{\Omega})} \leq\left\|y_{\epsilon_{1}}\left(\bar{v}_{\epsilon_{1}}\right)-y_{\epsilon_{2}}\left(\bar{v}_{\epsilon_{1}}\right)\right\|_{H_{0}^{1}(\Omega) \cap \mathcal{C}(\bar{\Omega})}+\left\|y_{\epsilon_{2}}\left(\bar{v}_{\epsilon_{1}}-\bar{v}_{\epsilon_{2}}\right)\right\|_{H_{0}^{1}(\Omega) \cap \mathcal{C}(\bar{\Omega})} \leq c(\mathcal{D})\left|\epsilon_{1}-\epsilon_{2}\right|,
$$
for all $\epsilon_{1}, \epsilon_{2} \in \mathcal{D}$.

Corollary 2.2. The mapping $\epsilon \mapsto \bar{u}_{\epsilon}$ is Lipschitz-continuous from $\mathcal{D}$ to $L^{2}(\Omega)$.

Proof. The assertion is immediate since, by construction, $\bar{u}_{\epsilon}=\frac{1}{\epsilon}\left(\bar{v}_{\epsilon}-\bar{y}_{\epsilon}\right)$ for all $\epsilon>0$.

Clearly, Corollaries 2.1 and 2.2 further imply the following result.

Corollary 2.3. The mapping $\epsilon \mapsto p_{\epsilon}$ is Lipschitz-continuous from $\mathcal{D}$ to $H_{0}^{1}(\Omega) \cap \mathcal{C}(\bar{\Omega})$.

Proof. Utilizing (1.3) in (1.2) and exploiting the Lipschitz continuity of $\bar{u}_{\epsilon}$ and $\bar{y}_{\epsilon}$, respectively, yields the assertion. 


\subsection{Parameter sensitivity}

We continue our investigation by studying the mapping $\epsilon \mapsto\left(\bar{y}_{\epsilon}, p_{\epsilon}\right)$. Under a strict complementary assumption, we establish the differentiability of this mapping with respect to $\epsilon$. First of all, we define the following operators:

$$
\begin{aligned}
g_{a}: \mathcal{D} \rightarrow \mathcal{C}(\bar{\Omega}), \quad g_{a}(\epsilon)=\frac{1}{\epsilon} p_{\epsilon}-\frac{\alpha}{\epsilon^{2}} \bar{y}_{\epsilon}+\frac{\alpha}{\epsilon^{2}} y_{a}, \\
g_{b}: \mathcal{D} \rightarrow \mathcal{C}(\bar{\Omega}), \quad g_{b}(\epsilon)=-\frac{1}{\epsilon} p_{\epsilon}+\frac{\alpha}{\epsilon^{2}} \bar{y}_{\epsilon}-\frac{\alpha}{\epsilon^{2}} y_{b} .
\end{aligned}
$$

Note that $g_{a}$ and $g_{b}$ appear in the maximum operators in (1.8)-(1.9):

$$
\left\{\begin{array}{l}
\mu_{\epsilon}^{a}=\max \left(0, \frac{1}{\epsilon} p_{\epsilon}+\frac{\alpha}{\epsilon^{2}}\left(y_{a}-\bar{y}_{\epsilon}\right)\right)=\max \left(0, g_{a}(\epsilon)\right), \\
\mu_{\epsilon}^{b}=\max \left(0,-\frac{1}{\epsilon} p_{\epsilon}+\frac{\alpha}{\epsilon^{2}}\left(\bar{y}_{\epsilon}-y_{b}\right)\right)=\max \left(0, g_{b}(\epsilon)\right) .
\end{array}\right.
$$

Therefore, in order to study the dependence of the Lagrange multipliers $\mu_{\epsilon}^{a}, \mu_{\epsilon}^{b}$ on the regularization parameter $\epsilon$, we need to consider the operators $g_{a}, g_{b}$. Suppose that $\epsilon \in \mathcal{D}$ is arbitrarily fixed. Then the Lipschitz-continuity of the mapping $\epsilon \mapsto\left(\bar{u}_{\epsilon}, \bar{y}_{\epsilon}, p_{\epsilon}\right)$ from $\mathcal{D}$ to $L^{2}(\Omega) \times H_{0}^{1}(\Omega) \times H_{0}^{1}(\Omega)$ ensures the existence of a weak accumulation point $\left(\dot{u}_{\epsilon}, \dot{y}_{\epsilon}, \dot{p}_{\epsilon}\right) \in L^{2}(\Omega) \times H_{0}^{1}(\Omega) \times H_{0}^{1}(\Omega)$ of

$$
\left(\frac{\bar{u}_{\epsilon_{n}}-\bar{u}_{\epsilon}}{\epsilon_{n}-\epsilon}, \frac{\bar{y}_{\epsilon_{n}}-\bar{y}_{\epsilon}}{\epsilon_{n}-\epsilon}, \frac{p_{\epsilon_{n}}-p_{\epsilon}}{\epsilon_{n}-\epsilon}\right)
$$

as $\epsilon_{n} \rightarrow \epsilon \in \mathcal{D}$ for $n \rightarrow \infty$. Further,

$$
\begin{aligned}
\frac{1}{\epsilon_{n}-\epsilon}\left(g_{a}\left(\epsilon_{n}\right)-g_{a}(\epsilon)\right)= & \frac{1}{\epsilon_{n}-\epsilon}\left(\frac{1}{\epsilon_{n}} p_{\epsilon_{n}}-\frac{\alpha}{\epsilon_{n}^{2}} \bar{y}_{\epsilon_{n}}+\frac{\alpha}{\epsilon_{n}^{2}} y_{a}-\frac{1}{\epsilon} p_{\epsilon}+\frac{\alpha}{\epsilon^{2}} \bar{y}_{\epsilon}-\frac{\alpha}{\epsilon^{2}} y_{a}\right) \\
= & \frac{1}{\epsilon_{n}-\epsilon}\left(\frac{1}{\epsilon}\left(p_{\epsilon_{n}}-p_{\epsilon}\right)-\left(\frac{1}{\epsilon}-\frac{1}{\epsilon_{n}}\right) p_{\epsilon_{n}}+\left(\frac{\alpha}{\epsilon^{2}}-\frac{\alpha}{\epsilon_{n}^{2}}\right) \bar{y}_{\epsilon_{n}}\right. \\
& \left.-\frac{\alpha}{\epsilon^{2}}\left(\bar{y}_{\epsilon_{n}}-\bar{y}_{\epsilon}\right)-\left(\frac{\alpha}{\epsilon^{2}}-\frac{\alpha}{\epsilon_{n}^{2}}\right) y_{a}\right) \\
= & \frac{1}{\epsilon} \frac{p_{\epsilon_{n}}-p_{\epsilon}}{\epsilon_{n}-\epsilon}-\frac{1}{\epsilon \epsilon_{n}} p_{\epsilon_{n}}+\frac{\alpha\left(\epsilon_{n}+\epsilon\right)}{\epsilon^{2} \epsilon_{n}^{2}} \bar{y}_{\epsilon_{n}}-\frac{\alpha}{\epsilon^{2}} \frac{\bar{y}_{\epsilon_{n}}-\bar{y}_{\epsilon}}{\epsilon_{n}-\epsilon}-\frac{\alpha\left(\epsilon_{n}+\epsilon\right)}{\epsilon^{2} \epsilon_{n}^{2}} y_{a}
\end{aligned}
$$

has a weak accumulation

$$
\dot{g}_{a}(\epsilon)=\frac{1}{\epsilon} \dot{p}_{\epsilon}-\frac{1}{\epsilon^{2}} p_{\epsilon}+\frac{2 \alpha}{\epsilon^{3}} \bar{y}_{\epsilon}-\frac{\alpha}{\epsilon^{2}} \dot{y}_{\epsilon}-\frac{2 \alpha}{\epsilon^{3}} y_{a}
$$

in $H_{0}^{1}(\Omega)$ as $\epsilon_{n} \rightarrow \epsilon$ for $n \rightarrow \infty$. Analogously, there exists a weak accumulation point of $\frac{1}{\epsilon_{n}-\epsilon}\left(g_{b}\left(\epsilon_{n}\right)-g_{b}(\epsilon)\right)$, which is given by

$$
\dot{g}_{b}(\epsilon)=-\frac{1}{\epsilon} \dot{p}_{\epsilon}+\frac{1}{\epsilon^{2}} p_{\epsilon}-\frac{2 \alpha}{\epsilon^{3}} \bar{y}_{\epsilon}+\frac{\alpha}{\epsilon^{2}} \dot{y}_{\epsilon}+\frac{2 \alpha}{\epsilon^{3}} y_{b} .
$$

By the compact embedding $H_{0}^{1}(\Omega) \subset L^{2}(\Omega)$ the accumulation points $\dot{g}_{a}(\epsilon)$ and $\dot{g}_{b}(\epsilon)$ are strong in $L^{2}(\Omega)$.

Without any further assumption, in general there is a need to distinguish between upper and lower accumulation points depending on whether $\epsilon_{n} \downarrow \epsilon$ or $\epsilon_{n} \uparrow \epsilon$, respectively. See [7] for a similar observation in a related context. However, under some type of strict complementarity condition this distinction is not required as all limits over $\epsilon_{n}$-sequences yield a unique accumulation point. This motivates the following assumption. 
Assumption 2.1. We assume that the solution of $\left(P_{\epsilon}\right)$ satisfies the strict complementarity condition

$$
\begin{aligned}
& \operatorname{meas}\left\{x \in \Omega \mid g_{a}(\epsilon)(x)=p_{\epsilon}(x)-\frac{\alpha}{\epsilon} \bar{y}_{\epsilon}(x)+\frac{\alpha}{\epsilon} y_{a}(x)=0 \text { a.e. }\right\}=0, \\
& \operatorname{meas}\left\{x \in \Omega \mid g_{b}(\epsilon)(x)=-p_{\epsilon}(x)+\frac{\alpha}{\epsilon} \bar{y}_{\epsilon}(x)-\frac{\alpha}{\epsilon} y_{b}(x)=0 \text { a.e. }\right\}=0 .
\end{aligned}
$$

Notice that by (1.8)-(1.9) the Lagrange multipliers for $\left(P_{\epsilon}\right)$ are given by

$$
\begin{aligned}
\mu_{\epsilon}^{a} & =\max \left(0, \frac{1}{\epsilon} p_{\epsilon}+\frac{\alpha}{\epsilon^{2}}\left(y_{a}-\bar{y}_{\epsilon}\right)\right)=g_{a}^{+}(\epsilon), \\
\mu_{\epsilon}^{b} & =\max \left(0,-\frac{1}{\epsilon} p_{\epsilon}+\frac{\alpha}{\epsilon^{2}}\left(\bar{y}_{\epsilon}-y_{b}\right)\right)=g_{b}^{+}(\epsilon),
\end{aligned}
$$

where $g_{a}^{+}=\max \left(0, g_{a}\right)$ and analogously for $g_{b}^{+}$. Hence, using (1.3), the condition (SC) requires that

$$
\operatorname{meas}\left\{x \in \Omega: \mu_{\epsilon}^{a}(x)+\gamma\left(y_{a}-\epsilon \bar{u}_{\epsilon}-\bar{y}_{\epsilon}\right)(x)=0\right\}=0,
$$

or equivalently, that the set where the max-operation is non-differentiable is of measure zero; analogously for the set involving $\mu_{\epsilon}^{b}$ and $y_{b}$.

Next we study the behavior of

$$
\frac{g_{a}^{+}\left(\epsilon_{n}\right)-g_{a}^{+}(\epsilon)}{\epsilon_{n}-\epsilon} \text { and } \frac{g_{b}^{+}\left(\epsilon_{n}\right)-g_{b}^{+}(\epsilon)}{\epsilon_{n}-\epsilon}
$$

as $\epsilon_{n} \rightarrow \epsilon$ for $n \rightarrow \infty$. For this purpose we introduce

$$
S_{a, \epsilon}:=\left\{x \in \Omega: g_{a}(\epsilon)(x)>0\right\}
$$

and analogously $S_{b, \epsilon}$. By $S_{a, \epsilon}^{c}$ and $S_{b, \epsilon}^{c}$ we denote the complement of $S_{a, \epsilon}$ and $S_{b, \epsilon}$ in $\Omega$, respectively. Recall that Assumption 2.1 implies meas $\left\{x \in \Omega: g_{a}(\epsilon)(x)=0\right\}=0$. For this reason, we have

$$
\text { meas } S_{a, \epsilon}=\operatorname{meas}\left\{x \in \Omega: g_{a}(\epsilon)(x)>0\right\}=\operatorname{meas}\left\{x \in \Omega: g_{a}(\epsilon)(x) \geq 0\right\}
$$

and analogously for $S_{b, \epsilon}$. Hence, for $v \in L^{2}(\Omega)$ we infer

$$
\begin{aligned}
\int_{\Omega}\left(\frac{g_{a}^{+}\left(\epsilon_{n}\right)-g_{a}^{+}(\epsilon)}{\epsilon_{n}-\epsilon}\right) v \mathrm{~d} x=\frac{1}{\epsilon_{n}-\epsilon} \int_{S_{a, \epsilon}^{c}} & \left(g_{a}^{+}\left(\epsilon_{n}\right)-g_{a}^{+}(\epsilon)\right) v \mathrm{~d} x \\
& +\frac{1}{\epsilon_{n}-\epsilon} \int_{S_{a, \epsilon}}\left(g_{a}^{+}\left(\epsilon_{n}\right)-g_{a}\left(\epsilon_{n}\right)\right) v \mathrm{~d} x+\frac{1}{\epsilon_{n}-\epsilon} \int_{S_{a, \epsilon}}\left(g_{a}\left(\epsilon_{n}\right)-g_{a}(\epsilon)\right) v \mathrm{~d} x .
\end{aligned}
$$

From the strict complementary condition (SC) and the definition of the set $S_{a, \epsilon}$ we obtain

$$
g_{a}(\epsilon)(x)<0 \text { for a.a. } x \in S_{a, \epsilon}^{c} \text { and } g_{a}(\epsilon)(x)>0 \text { for a.a. } x \in S_{a, \epsilon} .
$$

In addition, Corollaries 2.1 and 2.3 yield

$$
\lim _{n \rightarrow \infty} g_{a}\left(\epsilon_{n}\right)=g_{a}(\epsilon) \quad \text { in } \mathcal{C}(\bar{\Omega}) .
$$


The latter uniform convergence together with (2.14) results in

$$
\begin{array}{ll}
\lim _{n \rightarrow \infty} \frac{g_{a}^{+}\left(\epsilon_{n}\right)(x)-g_{a}^{+}(\epsilon)(x)}{\epsilon_{n}-\epsilon}=0 & \text { for a.a. } x \in S_{a, \epsilon}^{c}, \\
\lim _{n \rightarrow \infty} \frac{g_{a}^{+}\left(\epsilon_{n}\right)(x)-g_{a}\left(\epsilon_{n}\right)(x)}{\epsilon_{n}-\epsilon}=0 & \text { for a.a. } x \in S_{a, \epsilon} .
\end{array}
$$

Hence, for $n \rightarrow \infty$, the Lebesgue dominated convergence theorem and Assumption 2.1 yield

$$
\begin{aligned}
& \frac{1}{\epsilon_{n}-\epsilon} \int_{S_{a, \epsilon}^{c}}\left(g_{a}^{+}\left(\epsilon_{n}\right)-g_{a}^{+}(\epsilon)\right) v \mathrm{~d} x \rightarrow 0, \\
& \frac{1}{\epsilon_{n}-\epsilon} \int_{S_{a, \epsilon}}\left(g_{a}^{+}\left(\epsilon_{n}\right)-g_{a}\left(\epsilon_{n}\right)\right) v \mathrm{~d} x \rightarrow 0, \\
& \frac{1}{\epsilon_{n}-\epsilon} \int_{S_{a, \epsilon}}\left(g_{a}\left(\epsilon_{n}\right)-g_{a}(\epsilon)\right) v \mathrm{~d} x \rightarrow \int_{\Omega} \dot{g}_{a}(\epsilon) \chi_{S_{a, \epsilon}} v \mathrm{~d} x,
\end{aligned}
$$

where $\chi_{S_{a, \epsilon}}$ is the characteristic function of $S_{a, \epsilon}$. The analogous result holds true for $g_{b}^{+}$. These properties of $g_{a}^{+}, g_{b}^{+}$are important for the proof of the following theorem.

Theorem 2.2. Suppose that the solution of $\left(P_{\epsilon}\right)$ satisfies Assumption 2.1. Then the mappings $\mathbf{y}: \mathcal{D} \rightarrow L^{2}(\Omega)$, $\epsilon \mapsto \bar{y}_{\epsilon}$, and $\mathbf{p}: \mathcal{D} \rightarrow L^{2}(\Omega), \epsilon \mapsto p_{\epsilon}$, are strongly differentiable at $\epsilon \in \mathcal{D}$.

Proof. Let $(\delta u, \delta y, \delta p)$ be the difference of two weak accumulation points of

$$
\left(\frac{\bar{u}_{\epsilon_{n}}-\bar{u}_{\epsilon}}{\epsilon_{n}-\epsilon}, \frac{\bar{y}_{\epsilon_{n}}-\bar{y}_{\epsilon}}{\epsilon_{n}-\epsilon}, \frac{p_{\epsilon_{n}}-p_{\epsilon}}{\epsilon_{n}-\epsilon}\right)
$$

as $\epsilon_{n} \rightarrow \epsilon$. By $\delta \mu_{a}$ and $\delta \mu_{b}$ we denote the difference of the associated weak accumulation points of $\frac{g_{a}^{+}\left(\epsilon_{n}\right)-g_{a}^{+}(\epsilon)}{\epsilon_{n}-\epsilon}$ and $\frac{g_{b}^{+}\left(\epsilon_{n}\right)-g_{b}^{+}(\epsilon)}{\epsilon_{n}-\epsilon}$. Due to first order optimality, $\left(\delta u, \delta y, \delta p, \delta \mu^{a}, \delta \mu^{b}\right)$ is characterized by

$$
\begin{aligned}
& A \delta y=\delta u \quad \text { in } \Omega, \quad \delta y=0 \quad \text { on } \Gamma, \\
& A^{\star} \delta p=\delta y+\delta \mu^{b}-\delta \mu^{a} \quad \text { in } \Omega, \quad \delta p=0 \quad \text { on } \Gamma, \\
& \delta p+\alpha \delta u+\epsilon\left(\delta \mu^{b}-\delta \mu^{a}\right)=0 \\
& \delta \mu^{a}=\left(\frac{1}{\epsilon} \delta p-\frac{\alpha}{\epsilon^{2}} \delta y\right) \chi_{S_{a, \epsilon}} \\
& \delta \mu^{b}=\left(-\frac{1}{\epsilon} \delta p+\frac{\alpha}{\epsilon^{2}} \delta y\right) \chi_{S_{b, \epsilon}} .
\end{aligned}
$$

Inserting equations (2.18)-(2.20) in (2.16) and (2.17) and then rearranging terms, we obtain

$$
\begin{aligned}
A \delta y+\frac{1}{\epsilon}\left(\chi_{S_{a, \epsilon}}+\chi_{S_{b, \epsilon}}\right) \delta y & =\frac{1}{\alpha}\left(-I+\chi_{S_{a, \epsilon}}+\chi_{S_{b, \epsilon}}\right) \delta p \quad \text { in } \Omega, \quad \delta y=0 \quad \text { on } \Gamma \\
A^{\star} \delta p+\frac{1}{\epsilon}\left(\chi_{S_{a, \epsilon}}+\chi_{S_{b, \epsilon}}\right) \delta p & =\left(I+\frac{\alpha}{\epsilon^{2}} \chi_{S_{a, \epsilon}}+\frac{\alpha}{\epsilon^{2}} \chi_{S_{b, \epsilon}}\right) \delta y \quad \text { in } \Omega, \quad \delta p=0 \quad \text { on } \Gamma .
\end{aligned}
$$

Further, let us define the bilinear form associated with the differential operator $A$ :

$$
\mathbf{a}: H_{0}^{1}(\Omega) \times H_{0}^{1}(\Omega) \rightarrow \mathbb{R}, \quad \mathbf{a}(\eta, \tau)=\int_{\Omega}\left(\sum_{i, j=1}^{N} a_{i j}(x) D_{i} \eta(x) D_{j} \tau(x)\right) \mathrm{d} x .
$$


In view of $(2.21)-(2.22), \delta y, \delta p \in H_{0}^{1}(\Omega)$ are given by the solutions of

$$
\begin{array}{cl}
\mathbf{a}(\delta y, z)+\frac{1}{\epsilon}\left(\left(\chi_{S_{a, \epsilon}}+\chi_{S_{b, \epsilon}}\right) \delta y, z\right)_{L^{2}(\Omega)}=\left(\frac{1}{\alpha}\left(-I+\chi_{S_{a, \epsilon}}+\chi_{S_{b, \epsilon}}\right) \delta p, z\right)_{L^{2}(\Omega)} & \forall z \in H_{0}^{1}(\Omega), \\
\mathbf{a}(z, \delta p)+\frac{1}{\epsilon}\left(\left(\chi_{S_{a, \epsilon}}+\chi_{S_{b, \epsilon}}\right) \delta p, z\right)_{L^{2}(\Omega)}=\left(\left(I+\frac{\alpha}{\epsilon^{2}} \chi_{S_{a, \epsilon}}+\frac{\alpha}{\epsilon^{2}} \chi_{S_{b, \epsilon}}\right) \delta y, z\right)_{L^{2}(\Omega)} \quad \forall z \in H_{0}^{1}(\Omega) .
\end{array}
$$

Inserting $z=\delta p$ and $z=\delta y$ in (2.23) and (2.24), respectively, we find that

$$
\begin{aligned}
0 \geq\left(\frac{1}{\alpha}\left(-I+\chi_{S_{a, \epsilon}}+\chi_{S_{b, \epsilon}}\right) \delta p, \delta p\right)_{L^{2}(\Omega)} & =\mathbf{a}(\delta y, \delta p)+\frac{1}{\epsilon}\left(\left(\chi_{S_{a, \epsilon}}+\chi_{S_{b, \epsilon}}\right) \delta y, \delta p\right)_{L^{2}(\Omega)} \\
& =\mathbf{a}(\delta y, \delta p)+\frac{1}{\epsilon}\left(\left(\chi_{S_{a, \epsilon}}+\chi_{S_{b, \epsilon}}\right) \delta p, \delta y\right)_{L^{2}(\Omega)} \\
& =\left(\left(I+\frac{\alpha}{\epsilon^{2}} \chi_{S_{a, \epsilon}}+\frac{\alpha}{\epsilon^{2}} \chi_{S_{b, \epsilon}}\right) \delta y, \delta y\right)_{L^{2}(\Omega)} \\
& \geq 0 .
\end{aligned}
$$

From this we infer $\delta y=\delta p=0$, and hence by (2.18)-(2.19) $\delta u=\delta \mu_{a}=\delta \mu_{b}=0$. Thus, as $\epsilon_{n} \rightarrow \epsilon$,

$$
\left(\frac{\bar{y}_{\epsilon_{n}}-\bar{y}_{\epsilon}}{\epsilon_{n}-\epsilon}, \frac{p_{\epsilon_{n}}-p_{\epsilon}}{\epsilon_{n}-\epsilon}\right)
$$

has a unique weak accumulation point in $H_{0}^{1}(\Omega) \times H_{0}^{1}(\Omega)$. By the compact-embedding of $H_{0}^{1}(\Omega)$ in $L^{2}(\Omega)$, $\left(\frac{\bar{y}_{\epsilon_{n}}-\bar{y}_{\epsilon}}{\epsilon_{n}-\epsilon}, \frac{p_{\epsilon_{n}}-p_{\epsilon}}{\epsilon_{n}-\epsilon}\right)$ admits a unique accumulation point in $L^{2}(\Omega) \times L^{2}(\Omega)$. Finally, since our arguments hold for any convergent subsequences, a standard argument completes the proof.

Remark 2.1. Based on the optimality conditions, the derivatives $\mathbf{y}^{\prime}(\epsilon)$ and $\mathbf{p}^{\prime}(\epsilon)$ satisfy the following system:

$$
\begin{aligned}
& A \mathbf{y}^{\prime}(\epsilon)=\dot{u}_{\epsilon} \quad \text { in } \Omega, \quad \mathbf{y}^{\prime}(\epsilon)=0 \quad \text { on } \Gamma, \\
& A^{\star} \mathbf{p}^{\prime}(\epsilon)=\mathbf{y}^{\prime}(\epsilon)+\dot{\mu}_{b, \epsilon}-\dot{\mu}_{a, \epsilon} \quad \text { in } \Omega, \quad \mathbf{p}^{\prime}(\epsilon)=0 \quad \text { on } \Gamma, \\
& \mathbf{p}^{\prime}(\epsilon)+\alpha \dot{u}_{\epsilon}+\epsilon\left(\dot{\mu}_{b, \epsilon}-\dot{\mu}_{a, \epsilon}\right)=0 ; \\
& \dot{\mu}_{a, \epsilon}=\left(\frac{1}{\epsilon} \mathbf{p}^{\prime}(\epsilon)-\frac{1}{\epsilon^{2}} p_{\epsilon}+\frac{2 \alpha}{\epsilon^{3}} \bar{y}_{\epsilon}-\frac{\alpha}{\epsilon^{2}} \mathbf{y}^{\prime}(\epsilon)-\frac{2 \alpha}{\epsilon^{3}} y_{a}\right) \chi_{\mathcal{S}_{a, \epsilon} ;} \\
& \dot{\mu}_{b, \epsilon}=\left(-\frac{1}{\epsilon} \mathbf{p}^{\prime}(\epsilon)+\frac{1}{\epsilon^{2}} p_{\epsilon}-\frac{2 \alpha}{\epsilon^{3}} \bar{y}_{\epsilon}+\frac{\alpha}{\epsilon^{2}} \mathbf{y}^{\prime}(\epsilon)+\frac{2 \alpha}{\epsilon^{3}} y_{b}\right) \chi_{\mathcal{S}_{b, \epsilon}} .
\end{aligned}
$$

Remark 2.2. It is also of interest to study the case where Assumption 2.1 does not hold true. In this case, differentiability with respect to $\epsilon$ cannot be expected. However, the existence of weak accumulation points in $H_{0}^{1}(\Omega)$ of, e.g., $\bar{y}_{\epsilon_{n}}-\bar{y}_{\epsilon} /\left(\epsilon_{n}-\epsilon\right)$ for $\epsilon_{n} \rightarrow \epsilon$ still holds true by the Lipschitz property of $\bar{y}_{\epsilon}$ with respect to $\epsilon$ according to Corollary 2.1. On the other hand, one can no longer expect uniqueness of these weak accumulation points. In our numerics, the term on the right hand side of (3.8) then has to be interpreted as a difference approximation of the directional derivative of $\bar{y}_{\epsilon_{n}}$ in direction $\left(\epsilon_{n+1}-\epsilon_{n}\right)$.

\section{Extrapolation-BASED Algorithm}

Next we introduce a semismooth Newton (SSN) algorithm which utilizes the theoretical results of the previous section within an extrapolation framework. Conceptually, we exploit the differentiability property of the solution of $\left(P_{\epsilon}\right)$ in order to predict a solution of $\left(P_{\epsilon_{2}}\right)$ given an approximate solution of $\left(P_{\epsilon_{1}}\right)$ with $\epsilon_{1}>\epsilon_{2}$. Hence, 
the extrapolation serves the purpose of efficient initializations of the SSN-method for solving $\left(P_{\epsilon}\right)$ along a sequence of vanishing regularization parameters.

In Section 1.2 we provide an equivalent characterization of first order optimality by using a reformulation based on the max-function; compare (1.6)-(1.7). This reformulation is the starting point for the development of a rapidly convergent algorithm of SSN-type. For this purpose we need a generalized derivative for "linearizing" the max-operations in (1.6)-(1.7). Then we introduce the algorithm which, due to our choice of the generalized derivative, has the flavor of an active set strategy utilizing both the primal and the dual variables for identifying the active sets

$$
\begin{aligned}
& \overline{\mathcal{A}}_{\epsilon}^{a}:=\left\{x \in \Omega: \epsilon \bar{u}_{\epsilon}(x)+\bar{y}_{\epsilon}(x)=y_{a}(x)\right\}, \\
& \overline{\mathcal{A}}_{\epsilon}^{b}:=\left\{x \in \Omega: \epsilon \bar{u}_{\epsilon}(x)+\bar{y}_{\epsilon}(x)=y_{b}(x)\right\}
\end{aligned}
$$

at the optimal solution of $\left(P_{\epsilon}\right)$. Subsequently we rely on the following generalized derivative.

Definition 3.1. Let $X, Y$ be Banach spaces and $U$ be an open set in $X$. A mapping $F: U \rightarrow Y$ is said to be semismooth (or Newton differentiable) in $U$ if there exists a (possibly set-valued) mapping $\partial F: U \rightrightarrows \mathscr{L}(X, Y)$ such that

$$
\sup _{V \in \partial F(x+s)}\|F(x+s)-F(x)-V s\|_{Y}=o\left(\|s\|_{X}\right) \quad \text { as }\|s\|_{X} \rightarrow 0
$$

is satisfied for all $x \in U$. We call $\partial F$ the Newton differential, and its elements $V$ are referred to as Newton maps.

As shown in [5], a class of Newton maps for the maximum operator $\mathbb{M}(z)=\max (0, z)$ is given by

$$
\partial \mathbb{M}_{\xi}(z)(x)=\left\{\begin{array}{l}
1 \text { if } z(x)>0 \\
0 \text { if } z(x)<0 \\
\xi \text { if } z(x)=0
\end{array}\right.
$$

with arbitrarily fixed $\xi \in \mathbb{R}$, provided that $\mathbb{M}$ is defined as a mapping from $L^{q_{2}}(\Omega)$ to $L^{q_{1}}(\Omega)$ with $1 \leq q_{1}<q_{2}$; otherwise, i.e., for $q_{1}=q_{2}$ the mapping $\mathbb{M}$ is not Newton differentiable.

As pointed out earlier, the complementarity system can be equivalently expressed as

$$
\begin{aligned}
& \mu_{\epsilon}^{a}=\max \left(0, \frac{1}{\epsilon} p_{\epsilon}+\frac{\alpha}{\epsilon^{2}}\left(y_{a}-\bar{y}_{\epsilon}\right)\right) \\
& \mu_{\epsilon}^{b}=\max \left(0,-\frac{1}{\epsilon} p_{\epsilon}+\frac{\alpha}{\epsilon^{2}}\left(\bar{y}_{\epsilon}-y_{b}\right)\right)
\end{aligned}
$$

where $p_{\epsilon}$ solves the adjoint equation (1.2). Further note that the equalities in (3.5) have to hold in $L^{2}(\Omega)$, respectively, and $p_{\epsilon}, \bar{y}_{\epsilon} \in H_{0}^{1}(\Omega) \subset L^{q}(\Omega)$ with $q>2$ depending on the spatial dimension due to Sobolev embedding results. Hence, the max-operations are understood as mappings from $L^{q}(\Omega)$ to $L^{2}(\Omega)$ with $2<q$ which guarantees Newton-differentiability. Choosing $\xi=0$ for the Newton maps of the maximum operators in (3.5), we obtain the following algorithm for solving $\left(P_{\epsilon}\right)$; compare also [8].

Algorithm 3.1 (semismooth Newton method).

(i) Initialization: Choose initial data $p^{0}, y^{0} \in L^{2}(\Omega)$ and set $l=0$.

(ii) Determine the active and inactive sets:

$$
\begin{aligned}
& \mathcal{A}_{a}^{l}=\left\{x \in \Omega: \frac{1}{\epsilon} p^{l}(x)+\frac{\alpha}{\epsilon^{2}}\left(y_{a}(x)-y^{l}(x)\right)>0 \text { a.e. in } \Omega\right\} \\
& \mathcal{A}_{b}^{l}=\left\{x \in \Omega:-\frac{1}{\epsilon} p^{l}(x)+\frac{\alpha}{\epsilon^{2}}\left(y^{l}(x)-y_{b}(x)\right)>0 \text { a.e. in } \Omega\right\}, \\
& \mathcal{I}^{l}=\Omega \backslash\left(\mathcal{A}_{a}^{l} \cup \mathcal{A}_{b}^{l}\right) .
\end{aligned}
$$


(iii) Find the solution $\left(y^{l+1}, p^{l+1}, u^{l+1}, \mu_{a}^{l+1}, \mu_{b}^{l+1}\right)$ of

$$
\begin{aligned}
& A y^{l+1}=u^{l+1} \quad \text { in } \quad \Omega, \quad A^{\star} p^{l+1}=y^{l+1}-y_{d}-\mu_{a}^{l+1}+\mu_{b}^{l+1} \text { in } \Omega, \\
& y^{l+1}=0 \quad \text { on } \Gamma, \quad p^{l+1}=0 \quad \text { on } \Gamma \text {, } \\
& \alpha u^{l+1}+p^{l+1}+\epsilon\left(\mu_{b}^{l+1}-\mu_{a}^{l+1}\right)=0 \\
& \begin{array}{llllll}
\mu_{a}^{l+1}=\frac{1}{\epsilon} p^{l+1}+\frac{\alpha}{\epsilon^{2}}\left(y_{a}-y^{l+1}\right) & \text { on } \quad \mathcal{A}_{a}^{l}, \quad \mu_{a}^{l+1}=0 \quad \text { on } & \mathcal{I}^{l} \cup \mathcal{A}_{b}^{l} \\
\mu_{b}^{l+1}=-\frac{1}{\epsilon} p^{l+1}+\frac{\alpha}{\epsilon^{2}}\left(y^{l+1}-y_{b}\right) & \text { on } \quad \mathcal{A}_{b}^{l}, \quad \mu_{b}^{l+1}=0 \quad \text { on } & \mathcal{I}^{l} \cup \mathcal{A}_{a}^{l} .
\end{array}
\end{aligned}
$$

(iv) Stop, or set $l=l+1$ and go to (ii).

Typically, we terminate the algorithm by using the stopping rule: $\mathcal{A}_{a}^{n}=\mathcal{A}_{a}^{n-1}$ and $\mathcal{A}_{b}^{n}=\mathcal{A}_{b}^{n-1}$. For a local convergence analysis of SSN-methods of this type we refer to [5].

Fast convergence of the above algorithm hinges on the quality of the initial point. For this purpose, based on Theorem 2.2 we employ an extrapolation strategy with respect to $\epsilon$. We outline our approach next. Let $\left(\epsilon_{n}\right)$ be a sequence of regularization parameters given by

$$
\epsilon_{n+1}=\left(1-\kappa_{n}\right) \epsilon_{n} \quad \text { with } 0<\kappa_{n}<1
$$

Here, $\kappa_{n}$ is the reduction rate of the sequence $\left(\epsilon_{n}\right)$ of regularization parameters. By Theorem 2.2, the Taylor expansion of $\mathbf{y}$ at $\epsilon_{n+1}$ implies

$$
\mathbf{y}\left(\epsilon_{n+1}\right) \approx \mathbf{y}\left(\epsilon_{n}\right)+\mathbf{y}^{\prime}\left(\epsilon_{n}\right)\left(\epsilon_{n+1}-\epsilon_{n}\right)
$$

The derivative $\mathbf{y}^{\prime}\left(\epsilon_{n}\right)$ may be computed by solving the sensitivity equations (2.25)-(2.29). In order to save CPU-time we propose to use backward differences in $\epsilon$ to approximate $\mathbf{y}^{\prime}\left(\epsilon_{n}\right)$ :

$$
\mathbf{y}^{\prime}\left(\epsilon_{n}\right) \approx \frac{\mathbf{y}\left(\epsilon_{n}\right)-\mathbf{y}\left(\epsilon_{n-1}\right)}{\epsilon_{n}-\epsilon_{n-1}}
$$

Utilizing (3.8) in (3.7), we obtain

$$
\mathbf{y}\left(\epsilon_{n+1}\right) \approx \mathbf{y}\left(\epsilon_{n}\right)+\frac{\epsilon_{n+1}-\epsilon_{n}}{\epsilon_{n}-\epsilon_{n-1}}\left(\mathbf{y}\left(\epsilon_{n}\right)-\mathbf{y}\left(\epsilon_{n-1}\right)\right)
$$

Using (3.6), the optimal state $\mathbf{y}\left(\epsilon_{n+1}\right)=y_{n+1}$ of $\left(P_{\epsilon_{n+1}}\right)$ is approximated by

$$
y_{n+1} \approx y_{n+1}^{0}:=y_{n}+\frac{\kappa_{n}}{\kappa_{n-1}}\left(1-\kappa_{n-1}\right)\left(y_{n}-y_{n-1}\right)
$$

Similarly, the adjoint state $\mathbf{p}\left(\epsilon_{n+1}\right)=p_{n+1}$ at $\epsilon_{n+1}$ is approximated by

$$
p_{n+1} \approx p_{n+1}^{0}:=p_{n}+\frac{\kappa_{n}}{\kappa_{n-1}}\left(1-\kappa_{n-1}\right)\left(p_{n}-p_{n-1}\right)
$$

In this way, we extrapolate the solutions of $\left(P_{\epsilon_{n}}\right)$ and $\left(P_{\epsilon_{n-1}}\right)$ in order to get the initial guess $\left(y_{n+1}^{0}, p_{n+1}^{0}\right)$ for Algorithm 3.1 to solve the subsequent problem $\left(P_{\epsilon_{n+1}}\right)$. For $\epsilon_{0}$ we use $y^{0}=p^{0}=0$.

\section{NUMERICAL EXPERIMENTS}

Our goal in this section is to demonstrate the numerical reliability of our sensitivity-based extrapolation approach. As mentioned before and based on earlier experience $[10,11,13]$, due to the high sensitivity of $\left(P_{\epsilon}\right)$ with respect to $\epsilon$ and the corresponding challenge in its numerical solution for vanishing regularization parameter 

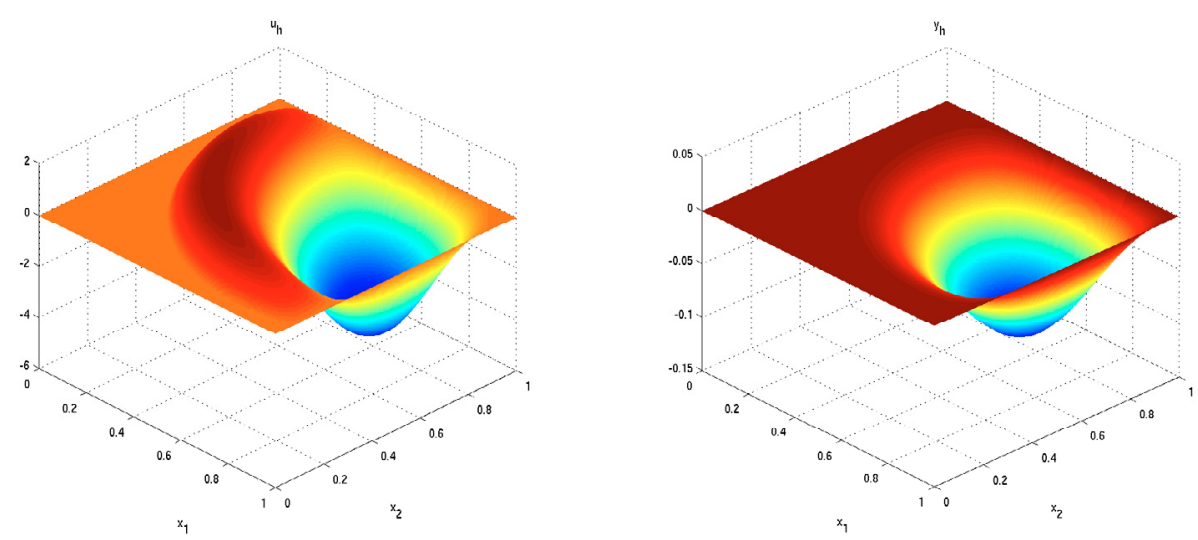

Figure 1. Computed optimal control $u_{h}$ (left plot) and corresponding state $y_{h}$ (right plot) for $\epsilon=10^{-6}$ and $h=1 / 256$.

the initialization along the $\epsilon$-sequence becomes an issue. It turns out that our initialization-by-extrapolation procedure achieves a significant speed up of Algorithm 3.1 compared to classical continuation strategies; compare, e.g., Tables 2 and 3 below.

In our numerical tests we use $\Omega=(0,1) \times(0,1)$ and $A=-\Delta$. The discretization of the problem is based on continuous piecewise linear finite elements on a uniform mesh, and the discrete versions of the active sets $\mathcal{A}_{a}$ and $\mathcal{A}_{b}$ are given by

$$
\begin{aligned}
& \left\{i: \frac{1}{\epsilon}\left(p_{h}\right)_{i}+\frac{\alpha}{\epsilon^{2}}\left(\left(y_{a, h}\right)_{i}-\left(y_{h}\right)_{i}\right)>0\right\}, \\
& \left\{i:-\frac{1}{\epsilon}\left(p_{h}\right)_{i}-\frac{\alpha}{\epsilon^{2}}\left(\left(y_{b, h}\right)_{i}-\left(y_{h}\right)_{i}\right)>0\right\}
\end{aligned}
$$

where the vectors $p_{h}, y_{h}, y_{a, h}, y_{b, h} \in \mathbb{R}^{N_{h}}$ are the values of the corresponding mesh functions at the nodal points of the discretization. Here, $h$ denotes the mesh size of discretization.

The numerical computations were carried out on a PC with a 250-GHz AMD processor and a 16-gigabyte memory.

\subsection{Example 1}

We choose:

$$
y_{d}=2 \cos \left(2 \pi x_{1} x_{2}\right), y_{b}=0, y_{a}=-1, \alpha=10^{-2}
$$

see Figure 1 for the numerical solution of $\left(P_{\epsilon}\right)$ with $\epsilon=10^{-6}$ and $h=1 / 256$.

First, in Table 1 we report on the results obtained by Algorithm 3.1 with simple initialization $y^{0}=p^{0}=0$, i.e., without the extrapolation strategy, in the case of vanishing regularization parameter. In addition to varying $\epsilon$, we provide the iteration numbers for various mesh sizes $h$. We clearly detect an unstable behavior of the algorithm with respect to $\epsilon$. Observing each column of Table 1, the number of iterations increases considerably as $\epsilon$ decreases. We also observe that small mesh sizes catalyze this adverse effect for small $\epsilon$. On the other hand, for large $\epsilon\left(\epsilon>10^{-4}\right.$ in our case) we even observe a mesh-independent behavior of the algorithm. This property was verified theoretically, also for a nonlinear case, in [8]. We point out that the results of [8] also hold true for small $\epsilon$, but in order to observe the mesh independence effect sufficiently small mesh sizes would be necessary.

Based on our numerical observations, the following reasons are responsible for the unstable behavior of the algorithm: First, the system of linear equations involved in each iteration of the algorithm is severely 
TABLE 1. Number of iterations required by Algorithm 3.1 for several choices of the regularization parameter $\epsilon$ and mesh sizes $h$.

\begin{tabular}{|c||c|c|c|c|}
\hline \multicolumn{1}{|c||}{} & \multicolumn{4}{c|}{ \# It. for various $h$} \\
\cline { 2 - 5 }$\epsilon$ & $1 / 32$ & $1 / 64$ & $1 / 128$ & $1 / 256$ \\
\hline \hline $10^{-1}$ & 4 & 4 & 4 & 4 \\
\hline $10^{-2}$ & 7 & 6 & 7 & 7 \\
\hline $10^{-3}$ & 14 & 14 & 15 & 15 \\
\hline $10^{-4}$ & 26 & 40 & 42 & 45 \\
\hline $10^{-5}$ & 32 & 59 & 109 & 156 \\
\hline $10^{-6}$ & 32 & 62 & 133 & 239 \\
\hline
\end{tabular}

TABLE 2. Results of the algorithm under a continuation strategy.

\begin{tabular}{|c||c|c|c|c|}
\hline \multicolumn{1}{|c||}{} & \multicolumn{4}{c|}{ \# It. for various $h$} \\
\cline { 2 - 5 }$\epsilon$ & $1 / 32$ & $1 / 64$ & $1 / 128$ & $1 / 256$ \\
\hline \hline $10^{-2}$ & 7 & 6 & 7 & 7 \\
\hline $10^{-3}$ & 8 & 9 & 10 & 10 \\
\hline $10^{-4}$ & 11 & 15 & 20 & 20 \\
\hline $10^{-5}$ & 6 & 11 & 19 & 25 \\
\hline $10^{-6}$ & 6 & 8 & 12 & 21 \\
\hline
\end{tabular}

ill-conditioned for small $\epsilon$. This effect becomes especially apparent on fine meshes. Secondly, the measure structure of the Lagrange multiplier associated with the upper bound complicates the numerical computation considerably. Figure 2 demonstrates that the structure of the multiplier becomes more and more singular as $\epsilon \rightarrow 0$.

The numerical experiments in [14] indicate that a (classical) continuation strategy may accelerate the convergence speed of the algorithm. Utilizing this concept, for our test problem we detect a remarkable speed-up. The basic idea of the continuation method is merely to use the solution of the regularization problem with a slightly larger regularization parameter as the initial data for the subsequent regularized problem (with a smaller parameter). Table 2 provides the results for this approach. Compared to the results in Table 1, the algorithm converges much faster. However, for the given mesh sizes we still experience the mesh-dependent behavior for fixed parameter $\epsilon \leq 10^{-4}$ as well as the unstable behavior with respect to decreasing $\epsilon$ for a fixed mesh size.

Now, we apply our sensitivity-based extrapolation method when solving the test problem numerically. We choose $\kappa_{n}$ constant by setting $\left(1-\kappa_{n}\right)=10^{-1}$ in (3.6). Thus, we obtain $\epsilon_{n}=10^{-1} \epsilon_{n-1}$. Initially we choose $\epsilon_{0}=10^{-2}$. We solve first $\left(P_{\epsilon_{1}}\right)$ utilizing the solution of $\left(P_{\epsilon_{0}}\right)$ as initial data. Subsequently, for all $n \geq 2$, $\left(P_{\epsilon_{n}}\right)$ is initialized by the extrapolation strategy, i.e., we utilize (3.10)-(3.11) for generating the initial data when solving $\left(P_{\epsilon_{n+1}}\right)$. In Table 3 we report on the numerical performance under this regime. Compared to the continuation strategy, we find that the initialization technique based on our extrapolation approach yields higher efficiency and stability with respect to the regularization parameter as well as the mesh size of discretization. Furthermore, observing Table 3, a rather mesh independent convergence of the algorithm is detected.

Instead of using the extrapolation strategy based on backward differences, one can also approximate the initial data by

$$
\begin{aligned}
y\left(\epsilon_{n+1}\right) \approx y^{0}\left(\epsilon_{n+1}\right) & :=y\left(\epsilon_{n}\right)+\mathbf{y}^{\prime}\left(\epsilon_{n}\right)\left(\epsilon_{n+1}-\epsilon_{n}\right), \\
p\left(\epsilon_{n+1}\right) \approx p^{0}\left(\epsilon_{n+1}\right) & :=p\left(\epsilon_{n}\right)+\mathbf{p}^{\prime}\left(\epsilon_{n}\right)\left(\epsilon_{n+1}-\epsilon_{n}\right),
\end{aligned}
$$



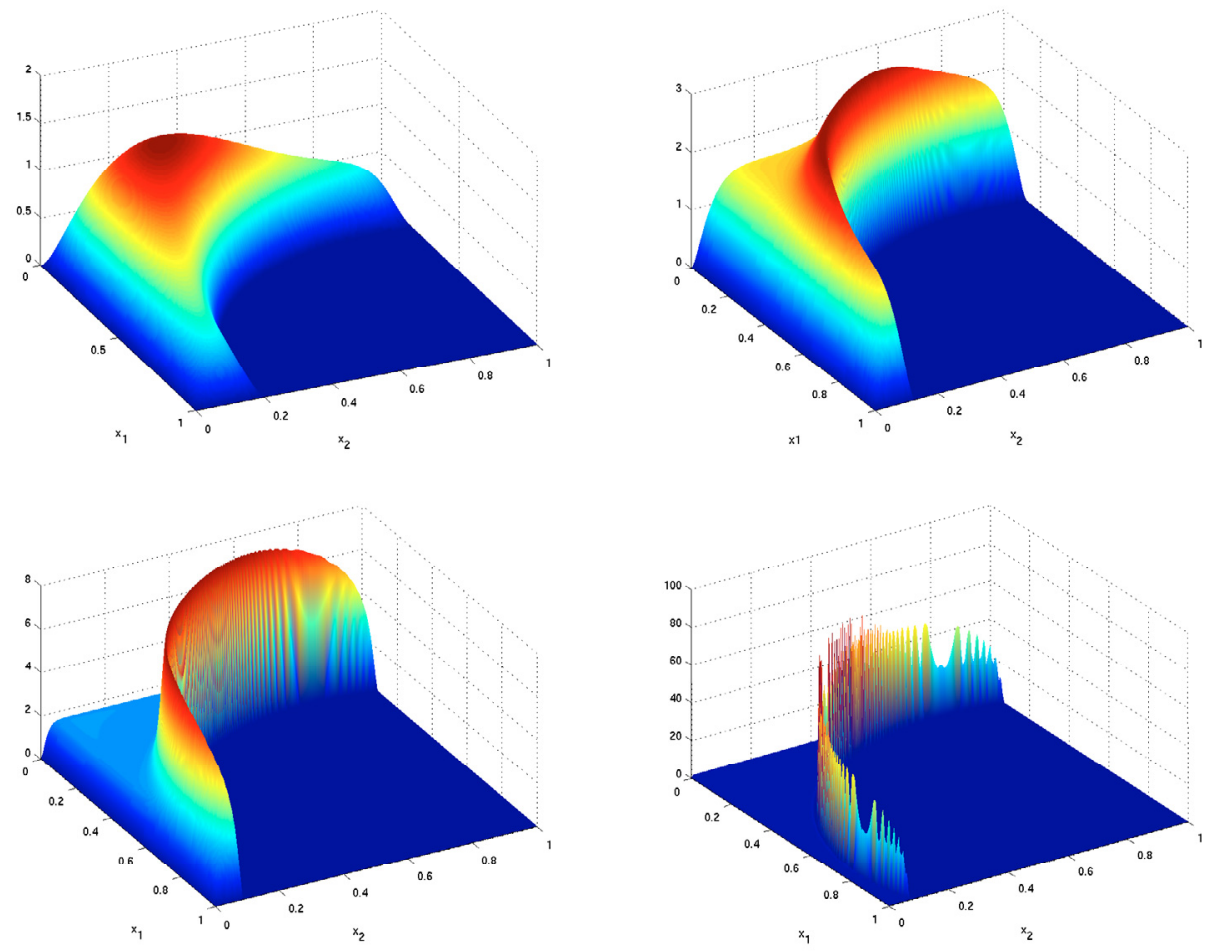

Figure 2. Computed Lagrange multipliers associated with the upper bound: Upper left: $\epsilon=$ $10^{-2}$, upper right: $\epsilon=10^{-3}$, lower left: $\epsilon=10^{-4}$ and lower right: $\epsilon=10^{-6}$.

TABLE 3. Results of the algorithm under the sensitivity-based extrapolation strategy and its stability.

\begin{tabular}{|c||c|c|c|c|}
\hline \multicolumn{1}{|c||}{} & \multicolumn{4}{c|}{ \# It. for various $h$} \\
\cline { 2 - 5 }$\epsilon$ & $1 / 32$ & $1 / 64$ & $1 / 128$ & $1 / 256$ \\
\hline \hline $10^{-2}$ & 7 & 6 & 7 & 7 \\
\hline $10^{-3}$ & 8 & 9 & 10 & 10 \\
\hline $10^{-4}$ & 4 & 6 & 6 & 6 \\
\hline $10^{-5}$ & 3 & 5 & 5 & 6 \\
\hline $10^{-6}$ & 6 & 5 & 6 & 7 \\
\hline
\end{tabular}

where the derivatives $\mathbf{y}^{\prime}\left(\epsilon_{n}\right)$ and $\mathbf{p}^{\prime}\left(\epsilon_{n}\right)$ are computed by solving the sensitivity equations (2.25)-(2.29); see Remark 2.1. In Table 4, we provide the corresponding numerical results with the choice $\epsilon_{n+1}=\epsilon_{n} \kappa$ and $\kappa=0.5$. The performance of this technique is satisfying as well. As before we observe a stabile behavior with respect to the regularization parameter as well as the mesh size $h$. Comparing the results with those obtained by the extrapolation strategy based on backward differences we conclude that the numerical performances of both methods are comparable. 
TABLE 4. Iteration numbers for computed sensitivities $\mathbf{y}^{\prime}\left(\epsilon_{n}\right)$ and $\mathbf{p}^{\prime}\left(\epsilon_{n}\right)$ with $\epsilon_{n+1}=0.5 \epsilon_{n}$.

\begin{tabular}{|c||c|c|c|c|c|}
\hline \multicolumn{1}{|c||}{$\epsilon$} & \multicolumn{5}{c|}{ \# It. for various $h$} \\
\cline { 2 - 6 } & $1 / 16$ & $1 / 32$ & $1 / 64$ & $1 / 128$ & $1 / 256$ \\
\hline \hline $10^{-2}$ & 5 & 7 & 6 & 7 & 7 \\
\hline $10^{-2} \kappa$ & 2 & 3 & 3 & 4 & 4 \\
\hline $10^{-2} \kappa^{2}$ & 2 & 2 & 3 & 4 & 4 \\
\hline $10^{-2} \kappa^{3}$ & 2 & 2 & 3 & 3 & 3 \\
\hline $10^{-2} \kappa^{4}$ & 2 & 3 & 3 & 3 & 3 \\
\hline $10^{-2} \kappa^{5}$ & 2 & 2 & 3 & 2 & 3 \\
\hline $10^{-2} \kappa^{6}$ & 3 & 2 & 2 & 2 & 3 \\
\hline $10^{-2} \kappa^{7}$ & 3 & 3 & 3 & 3 & 3 \\
\hline $10^{-2} \kappa^{8}$ & 4 & 3 & 4 & 4 & 3 \\
\hline $10^{-2} \kappa^{9}$ & 4 & 6 & 4 & 3 & 3 \\
\hline $10^{-2} \kappa^{10}$ & 4 & 6 & 4 & 4 & 3 \\
\hline
\end{tabular}

\subsection{Example 2}

Next we consider an example with an analytically known solution. For the optimal control, optimal state, the bounds and the cost parameter $\alpha$, we set

$$
\begin{aligned}
y_{\mathrm{opt}}\left(x_{1}, x_{2}\right) & =\sin \left(\pi x_{1}\right) \sin \left(\pi x_{2}\right), \\
u_{\mathrm{opt}}\left(x_{1}, x_{2}\right) & =-\Delta y_{\mathrm{opt}}\left(x_{1}, x_{2}\right)=2 \pi^{2} y_{\mathrm{opt}}\left(x_{1}, x_{2}\right), \\
y_{b}\left(x_{1}, x_{2}\right) & =\max \left(0.8 x_{2}+0.2, y_{\mathrm{opt}}\left(x_{1}, x_{2}\right)\right), \\
y_{a}\left(x_{1}, x_{2}\right) & =-3, \\
\alpha & =10^{-3} .
\end{aligned}
$$

The adjoint state at the optimal control is given by:

$$
p_{\text {opt }}\left(x_{1}, x_{2}\right)=-\alpha u_{\text {opt }}\left(x_{1}, x_{2}\right) .
$$

Furthermore, a few computations show that the multiplier for the upper bound

$$
\mu_{\mathrm{opt}}^{b}\left(x_{1}, x_{2}\right):=\left\{\begin{array}{lll}
10\left(0.8 x_{2}+0.2\right) & \text { if } \quad y_{\mathrm{opt}}\left(x_{1}, x_{2}\right) \geq 0.8 x_{2}+0.2 \\
0 & \text { if } y_{\mathrm{opt}}\left(x_{1}, x_{2}\right) \leq 0.8 x_{2}+0.2
\end{array}\right.
$$

satisfies the complementarity slackness conditions for $(P)$. For the Lagrange multiplier associated with the lower bound, we set $\mu_{\mathrm{opt}}^{a}=0$. Finally, from the adjoint state equation we obtain the desired state

$$
y_{d}=\Delta p_{\mathrm{opt}}+y_{\mathrm{opt}}+\mu_{\mathrm{opt}}^{b}
$$

All these quantities are depicted in Figure 3.

For comparison, for fixed mesh size $h=1 / 128$ in Table 5 we collect the numerical results obtained by our algorithm without a special initialization strategy. Additionally, we report on the $L^{2}$-error between the computed numerical solution and the analytical one. Upon studying Table 5 we note that the error stabilizes at a some level as $\epsilon$ tends to 0 . This clearly shows that, depending on the mesh size of discretization $h$, there is an $\epsilon(h)$ such that the error stabilizes for $\epsilon<\epsilon(h)$. In order to further reduce the error a reduction of $h$ is necessary. A further reduction of $\epsilon$ (for fixed $h$ ), on the other hand, only increases the ill-conditioning due to small regularization parameter but it does not reduce the overall error. The ill-conditioning is reflected by 

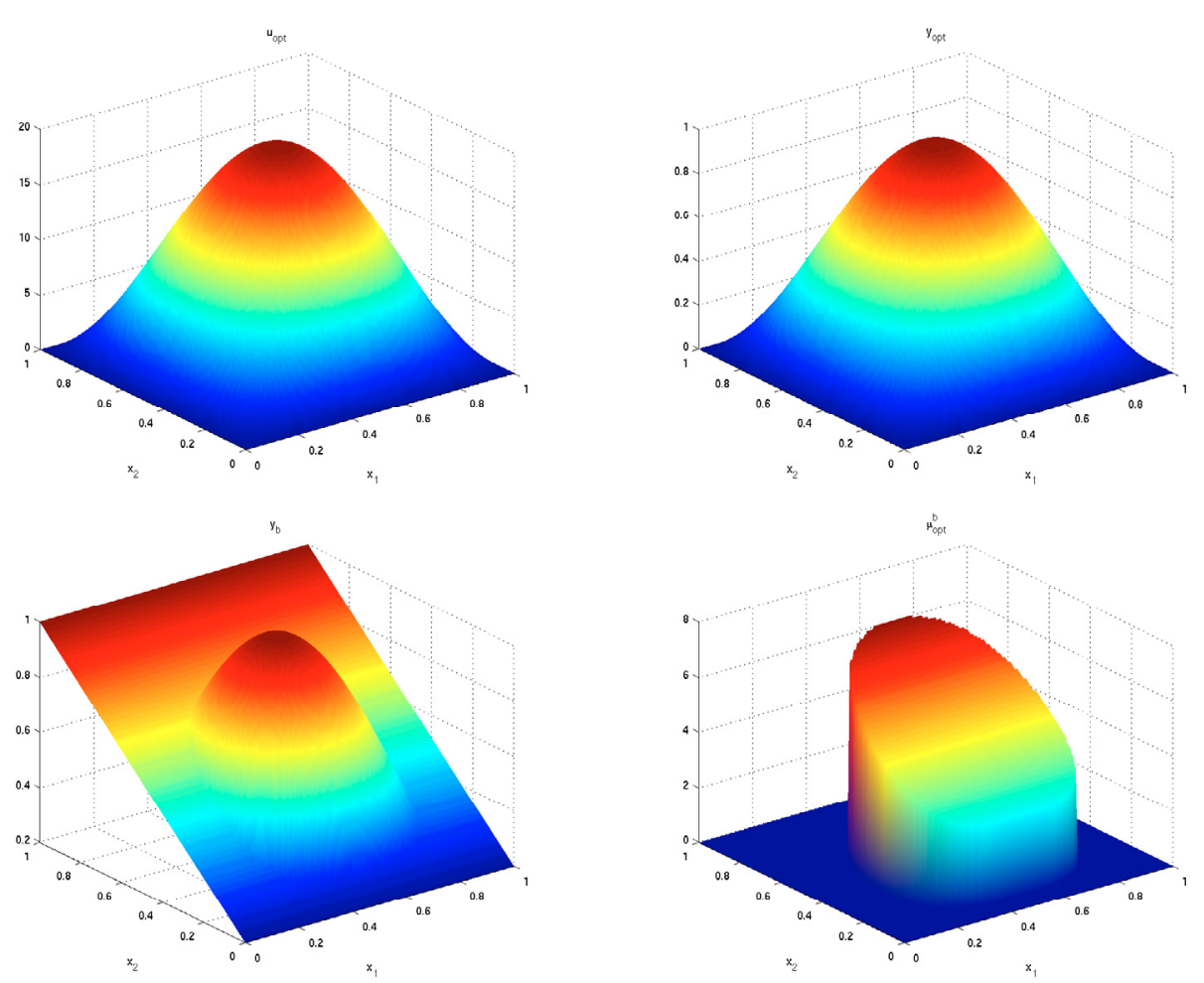

Figure 3. Example 2. Optimal control $u_{\text {opt }}$ (upper left), optimal state $y_{\text {opt }}$ (upper right), upper bound $y_{b}$ (lower left) and Lagrange multiplier associated with the upper bound $\mu_{\mathrm{opt}}^{b}$ (lower right).

TABLE 5. Numerical performance of Algorithm 3.1 with respect to decreasing regularization parameter.

\begin{tabular}{|c||c|c|c|c|c|}
\hline$\epsilon$ & $\#$ it. & $\left\|u_{h}^{\epsilon}-u_{\text {opt }}\right\|_{L^{2}(\Omega)}$ & $\left\|y_{h}^{\epsilon}-y_{\text {opt }}\right\|_{L^{2}(\Omega)}$ & $\left\|p_{h}^{\epsilon}-p_{\text {opt }}\right\|_{L^{2}(\Omega)}$ & $\left\|\mu_{h}^{b, \epsilon}-\mu_{\text {opt }}^{b}\right\|_{L^{2}(\Omega)}$ \\
\hline \hline $10^{-4.0}$ & 25 & $8.77393 \mathrm{e}-02$ & $8.85558 \mathrm{e}-04$ & $2.68096 \mathrm{e}-04$ & $2.36374 \mathrm{e}-01$ \\
\hline $10^{-5.0}$ & 42 & $4.95932 \mathrm{e}-03$ & $9.43736 \mathrm{e}-05$ & $2.91984 \mathrm{e}-05$ & $1.09244 \mathrm{e}-01$ \\
\hline $10^{-6.0}$ & 69 & $1.48140 \mathrm{e}-03$ & $1.05259 \mathrm{e}-05$ & $4.20660 \mathrm{e}-06$ & $2.27157 \mathrm{e}-02$ \\
\hline $10^{-7.0}$ & 70 & $1.60176 \mathrm{e}-03$ & $3.96104 \mathrm{e}-06$ & $1.84645 \mathrm{e}-06$ & $7.46035 \mathrm{e}-03$ \\
\hline $10^{-8.0}$ & 73 & $1.61771 \mathrm{e}-03$ & $3.78954 \mathrm{e}-06$ & $1.64142 \mathrm{e}-06$ & $8.01558 \mathrm{e}-03$ \\
\hline $10^{-9.0}$ & 71 & $1.61034 \mathrm{e}-03$ & $3.78286 \mathrm{e}-06$ & $1.62170 \mathrm{e}-06$ & $8.11886 \mathrm{e}-03$ \\
\hline
\end{tabular}

the increasing number of iterations until successful termination of the algorithm; see the second column from left.

Finally we study the effect of employing our new sensitivity-based initialization by extrapolation. The corresponding results are collected in Table 6. In the third column we find the relative CPU-time, i.e., the ratio of the CPU-time needed when using our extrapolation scheme vs. the CPU-time corresponding to the run reported on in Table 5. As before we observe a significant speed-up when using our initialization strategy especially in cases of small regularization parameters. The strong improvement for $\epsilon \leq 1.0 \mathrm{e}-8$ may be related to the high quality of the initial points. This can be seen from the forth and fifth column of Table 6 , where we show the $L^{2}$-distance of the optimal discrete state and adjoint state of the regularized problem to the corresponding 
TABLE 6. Speed up of Algorithm 3.1 under the extrapolation-initialization-strategy.

\begin{tabular}{|c||c|c|c|c|}
\hline$\epsilon$ & \#It. & CPU-ratio & $\left\|y_{h}^{\epsilon}-y_{h}^{\text {ext }, \epsilon}\right\|_{L^{2}(\Omega)}$ & $\left\|p_{h}^{\epsilon}-p_{h}^{\text {ext }, \epsilon}\right\|_{L^{2}(\Omega)}$ \\
\hline \hline $10^{-7}$ & 57 & 0.88 & $4.7052 \mathrm{e}-07$ & $1.9186 \mathrm{e}-07$ \\
\hline $10^{-8}$ & 5 & 0.09 & $6.9104 \mathrm{e}-09$ & $3.8095 \mathrm{e}-09$ \\
\hline $10^{-9}$ & 5 & 0.09 & $7.3953 \mathrm{e}-11$ & $4.4084 \mathrm{e}-11$ \\
\hline
\end{tabular}
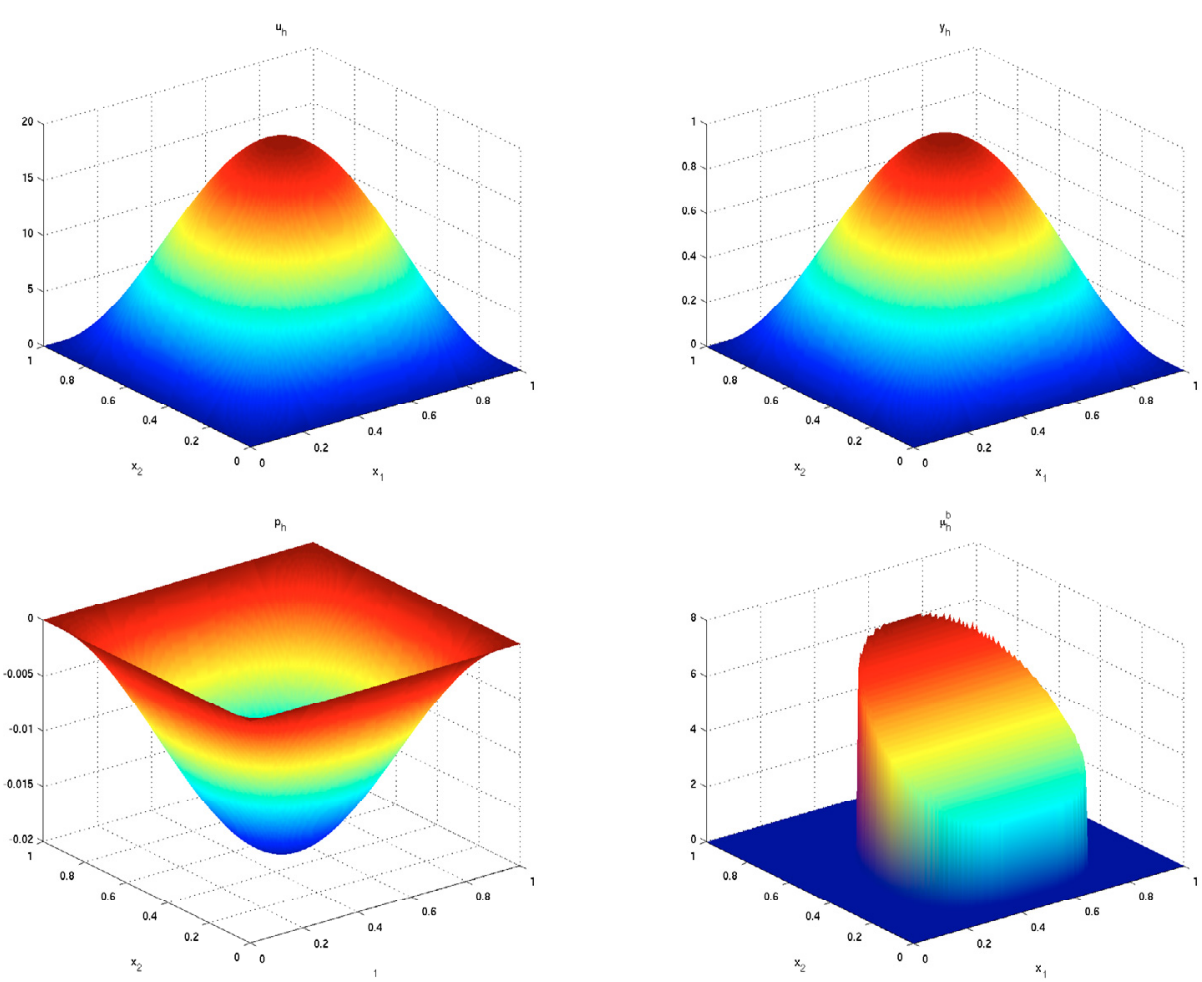

FiguRE 4. Computed numerical solution for $\epsilon=10^{-9}$ and $h=1 / 128$ : optimal control (upper left), Optimal state (upper right), adjoint state (lower left) and Lagrange multiplier associated with the upper bound (lower right).

initial data $y_{h}^{\text {ext }, \epsilon}$ and $p_{h}^{\text {ext }, \epsilon}$ when solving $\left(P_{\epsilon}\right)$. In Figure 4 we provide the graphs of the numerical solution for $\epsilon=1.0 \mathrm{e}-9$, which is close to the analytic solution; compare Figure 3.

\section{COnClusion}

Studying the sensitivity of the solution of the (Lavrentiev) regularized state constrained optimal control problem with respect to the regularization parameter is beneficial as it allows to employ highly efficient initialization schemes along a sequence of vanishing regularization parameters, i.e., in the case of convergence to the original problem. In our numerical test runs it turns out that this strategy successfully copes with the increasing ill-conditioning of the problems as $\epsilon \rightarrow 0$ and as $h$ is refined. Further, a combination with a nested iteration concept allows to even further increase the efficiency of the algorithm such that we observe a rather mesh independent behavior of our method when solving the state constrained optimal control problem. The sensitivity analysis, however, is also of interest in its own right as it allows to study the quality of the dependence of the regularized solution on the regularization parameter. Within path-following frameworks (see [6] for problems 
with low multiplier regularity, or [3] for a general account in the context of smooth Newton-based approaches), where the path $\left\{\left(\bar{u}_{\epsilon}, \bar{y}_{\epsilon}\right): \epsilon>0\right\}$ is induced by $\epsilon$, it is at the basis of studying further properties such as, e.g., the length of the path and other relevant quantities.

\section{REFERENCES}

[1] J.-J. Alibert and J.-P. Raymond, Boundary control of semilinear elliptic equations with discontinuous leading coefficients and unbounded controls. Numer. Funct. Anal. Optim. 3/4 (1997) 235-250.

[2] E. Casas, Control of an elliptic problem with pointwise state contraints. SIAM J. Contr. Opt. 4 (1986) 1309-1322.

[3] P. Deuflhard, Newton Methods for Nonlinear Problems. Affine Invariance and Adaptive Algorithms, Springer Series in Computational Mathematics 35. Springer-Verlag, Berlin (2004).

[4] M. Hintermüller, Mesh-independence and fast local convergence of a primal-dual active-set method for mixed control-state constrained elliptic control problems. ANZIAM Journal 49 (2007) 1-38.

[5] M. Hintermüller, K. Ito and K. Kunisch, The primal-dual active set strategy as a semismooth Newton method. SIAM J. Optim. 13 (2003) 865-888.

[6] M. Hintermüller and K. Kunisch, Feasible and non-interior path-following in constrained minimization with low multiplier regularity. SIAM J. Control Optim. 45 (2006) 1198-1221.

[7] M. Hintermüller and K. Kunisch, Path-following methods for a class of constrained minimization problems in function space. SIAM J. Optim. 17 (2006) 159-187.

[8] M. Hintermüller, F. Tröltzsch and I. Yousept, Mesh-independence of semismooth Newton methods for Lavrentiev-regularized state constrained nonlinear optimal control problems. Numer. Math. 108 (2008) 571-603.

[9] M. Hinze and C. Meyer, Variational discretization of Lavrentiev-regularized state constrained elliptic optimal control problems. Computat. Optim. Appl. (2009), doi: 10.1007/s10589-008-9198-1.

[10] C. Meyer, A. Rösch and F. Tröltzsch, Optimal control of PDEs with regularized pointwise state constraints. Comp. Optim. Appl. 33 (2006) 209-228.

[11] C. Meyer, U. Prüfert and F. Tröltzsch, On two numerical methods for stat e-constrained elliptic control problems. Optim. Method. Softw. 22 (2007) 871-899.

[12] F. Tröltzsch, Regular Lagrange multipliers for control problems with mixed pointwise control-state constraints. SIAM J. Optim. 15 (2004/2005) 616-634 (electronic).

[13] F. Tröltzsch and I. Yousept, A regularization method for the numerical solution of elliptic boundary control problems with pointwise state constraints. Comp. Optim. Control 42 (2009) 43-63.

[14] I. Yousept, Vergleich von Lösungsverfahren zur Behandlung elliptischer Optimalsteuerungsprobleme. Master's thesis, TU-Berlin, Germany (2005). 